\title{
Some recent advances in the theory of homogeneous isotropic turbulence
}

\author{
SUJAN K DHAR, ANIRBAN SAIN, ASHWIN PANDE and RAHUL PANDIT* \\ Department of Physics, Indian Institute of Science, Bangalore 560012, India \\ *Also at Jawaharlal Nehru Centre for Advanced Scientific Research, Bangalore, India
}

\begin{abstract}
We review some advances in the theory of homogeneous, isotropic turbulence. Our emphasis is on the new insights that have been gained from recent numerical studies of the threedimensional Navier Stokes equation and simpler shell models for turbulence. In particular, we examine the status of multiscaling corrections to Kolmogorov scaling, extended self similarity, generalized extended self similarity, and non-Gaussian probability distributions for velocity differences and related quantities. We recount our recent proposal of a wave-vector-space version of generalized extended self similarity and show how it allows us to explore an intriguing and apparently universal crossover from inertial- to dissipation-range asymptotics.
\end{abstract}

Keywords. Isotropic turbulence; homogeneous turbulence; turbulence simulation and modelling; theory and models of chaotic systems; critical point phenomena.

PACS Nos $47.27 ; 05.45 ; 05.70$

\section{Introduction}

If a fluid is forced through a grid (say in a wind tunnel) or through an orifice, the flow downstream is turbulent [1]. Most of the energy is contained in the large scales of the flow; transport too is dominated by the large scales. Nevertheless, there has been great interest in the study of turbulence at small scales; these include the dissipation range, in which energy is dissipated, and the inertial range, that lies in between dissipation and large scales. There are both practical and fundamental reasons for this continuing interest. We concentrate on the latter here and refer the reader to the recent article by Sreenivasan and Antonia [2] for a more detailed discussion. Other recent reviews include those by Siggia [3] and Nelkin [4], the brief overview by L'vov and Procaccia [5], and the books by McComb [6], Frisch [7], and Bohr, Jensen, Paladin, and Vulpiani [8]; older material can be found in the classics by Batchelor [9], Tennnekes and Lumley [10], and Monin and Yaglom [11].

There are many fundamental reasons for studying the statistical properties of smallscale turbulence, which is (in many cases, e.g., far from boundaries, etc.), to a good approximation [2,12,13], homogeneous and isotropic: (1) These properties are believed to be universal, i.e., independent of the details of the flow; specifically, the asymptotic behaviours of certain correlation functions (the structure functions defined below) are universal in a way that is reminiscent of, but perhaps richer than, the universality of correlation functions at continuous phase transitions [14]. (2) From the point of view of 


\section{Sujan $K$ Dhar et al}

dynamical systems, turbulence is the quintessential example of spatiotemporal chaos [8] in an extended, deterministic, driven system. (3) To the extent that the chaos generated by turbulence can be mimicked by an external noise [15], one can try to obtain the properties of correlation functions by studying effective stochastic equations, which are amenable to analytical studies that use renormalization-group, field-theoretical, or closure techniques [16-20]. (4) And last, the scale-invariance of correlation functions in the inertial range is an example of self-organized criticality [21], though very little has been gained by thinking about turbulence in this manner.

This paper is organized as follows: Section 2 gives a brief, elementary overview of the phenomenology of homogeneous, isotropic turbulence to establish notations. Section 3 introduces some of the deterministic models that have been used in theoretical and numerical work and reviews what has emerged from such studies. We make some concluding remarks in $\S 4$.

\section{The phenomenology of homogeneous isotropic turbulence}

Experiments on turbulent flows often measure the velocity $\mathbf{v}(\mathbf{r}, t)$, or the energy dissipation $\epsilon$ at a given spatial point $\mathbf{r}$ at time $t$, by using probes such as hot-wire anemometers. These are also augmented by flow-visualisation measurements [1]. Therefore, a typical experiment yields a time series for $\mathbf{v}$ or $\epsilon$ at a given spatial point. Most experiments are done with a mean background flow with velocity $U$ and the resulting temporal information is converted into spatial information by using Taylor's frozen-flow hypothesis: This states that the turbulence is advected by the mean flow without significant distortion, so a temporal delay $\Delta t$ can be converted into a spatial separation (along the mean flow) $\Delta x=\Delta t U$ to obtain spatial correlation functions. Such a hypothesis is reasonable when $|\mathrm{U}| \gg v_{\text {rms }}$, the root-mean-square fluctuations in the velocity because of the turbulence; for a more critical evaluation of its validity we refer the reader to refs $[2,4,7]$. The Reynolds number $\operatorname{Re}=U L / \nu$ is the relevant control parameter, which, when large, leads to turbulence; here $U$ and $L$ are typical velocity and length scales, respectively, and $\nu$ is the kinematic viscosity. The Taylor-microscale Reynolds number $\operatorname{Re}_{\lambda}$, which is useful in comparing widely different systems, will be defined in $\S 3$.

Another important approximation is made in obtaining the energy dissipation $\epsilon \equiv \frac{\nu}{2} \sum_{i j}\left(\partial_{i} \mathbf{v}_{j}+\partial_{j} \mathbf{v}_{i}\right)^{2}$, where the subscripts $i$ and $j$ denote Cartesian components and $\nu$ is the kinematic viscosity: It turns out to be difficult to measure $\epsilon$, so, typically, the surrogate $\epsilon^{\prime} \sim\left(\partial u_{1} / \partial t\right)^{2}$ is measured, with $u_{1}$ the velocity in the streamwise direction; again we recommend [2] for a discussion of the validity of using this approximation.

Given such data, a variety of information can be extracted about the turbulent flow in question. We concentrate on measurements that can be classified into three rough categories:

1. The first is predominantly qualitative information about coherent structures, such as vortex streets, eddies, etc. These are helpful, as we will see below, in developing physically appealing scenarios for turbulent cascades, intermittency, and multifractal dissipation.

2. The second is the measurement of spatial correlation functions (typically via the Taylor hypothesis), the most important ones being the order- $p$ velocity structure

Pramana - J. Phys., Vol. 48, No. 1, January 1997 (Part I) 
functions,

$$
\mathcal{S}_{p}(r) \equiv\left\langle\left[\mathbf{v}_{i}(\mathbf{x}+\mathbf{r})-\mathbf{v}_{i}(\mathbf{x})\right]^{p}\right\rangle .
$$

The angular brackets imply, in principle, a spatiotemporal average over the statistical steady state that obtains in the turbulent fluid. In experiments, often only the component parallel to the mean flow is used. Some workers use a modulus instead of the square brackets in (1), but others [2] discourage its use; it clearly makes no difference for even $p$ but can affect exponents. [22] for odd $p$. Other commonly measured correlation functions include the energy spectrum $E(k) \equiv 4 \pi k^{2}\left\langle|\mathbf{v}(\mathbf{k})|^{2}\right\rangle$, where $\mathbf{v}(\mathbf{k})$ is the spatial Fourier transform of the velocity, $\mathbf{k}$ the wave vector, and $k \equiv|\mathbf{k}|$.

3. The third is the measurement of probability distribution functions for velocity differences, such as $\delta \mathbf{v}_{i} \equiv\left[\mathbf{v}_{i}(\mathbf{x}+\mathbf{r})-\mathbf{v}_{i}(\mathbf{r})\right]$ (in experiments $i$ is often the direction of the mean flow), the energy dissipation $\epsilon$, etc.

\subsection{Flow visualization}

The first category of measurements, namely, flow visualization, have shown that fluid turbulence is typically associated with coherent structures. These can be eddies, as in mixing-layer experiments, or plumes, as in Rayleigh-Béenard systems, etc. [1]. These structures, though clear to the eye, are difficult to quantify and define. In recent elegant experiments $[23,24,25]$ high-vorticity, low-pressure filaments, of the type seen in direct numerical simulations $(\S 3)$, have been visualized directly in different turbulent flows.

Many years ago Richardson [26] suggested the following cascade picture: Turbulent flow is considered to be made up of a hierarchy of eddies (disturbances or inhomogeneities in the flow) of various length scales. Energy is added to the turbulence in the integral range, which corresponds to large length scales or small wave numbers, via large-scale processes like stirring. The nonlinear interactions in the fluid cause this energy to flow towards small scales (large wave numbers), by the formation of successively smaller eddies: large eddies lose stability, form smaller eddies, which in turn lose stability, break into even smaller eddies, and so on. In this process, the energy cascades from large to small length scales, with negligible energy dissipation in the inertial range. Viscous processes lead to significant dissipation once the sizes of eddies become comparable to, or smaller than, the dissipation scale $\eta_{\mathrm{d}} \equiv\left(\nu^{3} /\langle\epsilon\rangle\right)^{1 / 4}$.

\subsection{Structure functions}

If the Reynolds number $\operatorname{Re}$ is sufficiently large then there is a substantial inertial range, in which there can be universal scaling behaviour as noted first by Kolmogorov [27] in 1941 (henceforth $\mathrm{K} 41$ ). His theory is based on the following premises:

1. On small scales and far away from boundaries fluctuations in a turbulent flow are homogeneous and isotropic, statistically steady, and universally determined by the mean energy dissipation rate (per unit mass of fluid) $\epsilon$ and the kinematic viscosity $\nu$. 


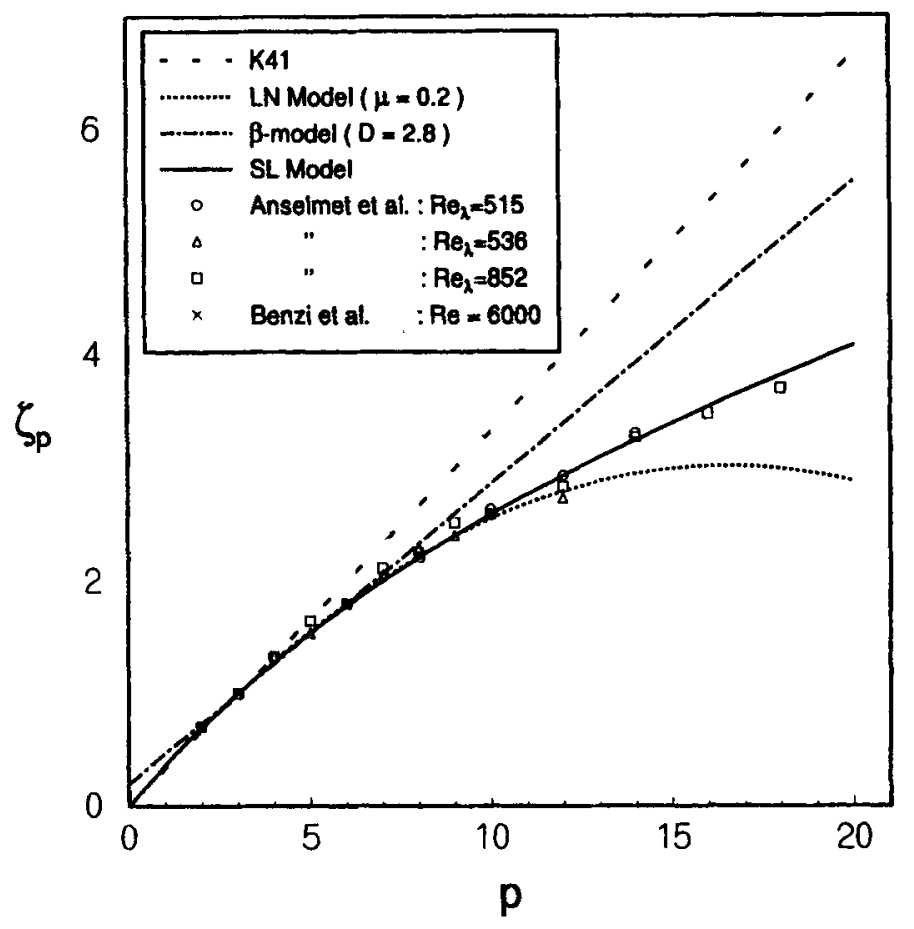

Figure 1. A plot comparing data for the inertial-range exponent $\zeta_{p}$ with different theoretical predictions. Experimental data are from Anselmet et al (1984) and Benzi et al (1993). Theoretical predictions are from K41, the Kolmogorov log-normal model [42], with $\mu=0.2$, the $\beta$ model (with $D=2.8$ ), and the SL model (see text). Clearly the SL prediction agrees best with experimental data.

2. In the inertial range, viscosity does not play a significant part, so the statistical properties at a given length scale $\ell$ are determined by $\epsilon$ (which is finite, and non-zero) and the length scale $\ell$ itself.

Given these assumptions, correlation functions assume simple and universal scaling forms in the inertial range, which follow via dimensional analysis. (Note that the dimensions of $\epsilon$ and the energy spectrum $E(k)$, which measures the distribution of energy over different length scales, are, respectively, $\mathrm{L}^{2} \mathrm{~T}^{-3}$ and $\mathrm{L}^{3} \mathrm{~T}^{-2}$.) Specifically

$$
\mathcal{S}_{p}(r) \approx C_{p}(\langle\epsilon\rangle r)^{p / 3}
$$

where $C_{p}$ are universal amplitudes; the case $p=3$ yields the Kolmogorov 4/5 law $\left(C_{3}=-4 / 5\right)$. Also

$$
E(k)=C\langle\epsilon\rangle^{2 / 3} k^{-5 / 3},
$$

which is the celebrated $-5 / 3$ law.

The second category of experimental measurements comprise studies of such structure functions. They find that low-order structure functions $(p \leq 3)$ are in close agreement with the predictions of $\mathrm{K} 41$ [28]. In particular, the $-5 / 3$ law has been checked [2,13,29] over several decades of wavenumber $k$; the Kolmogorov constant is found to be $[2,30]$ 


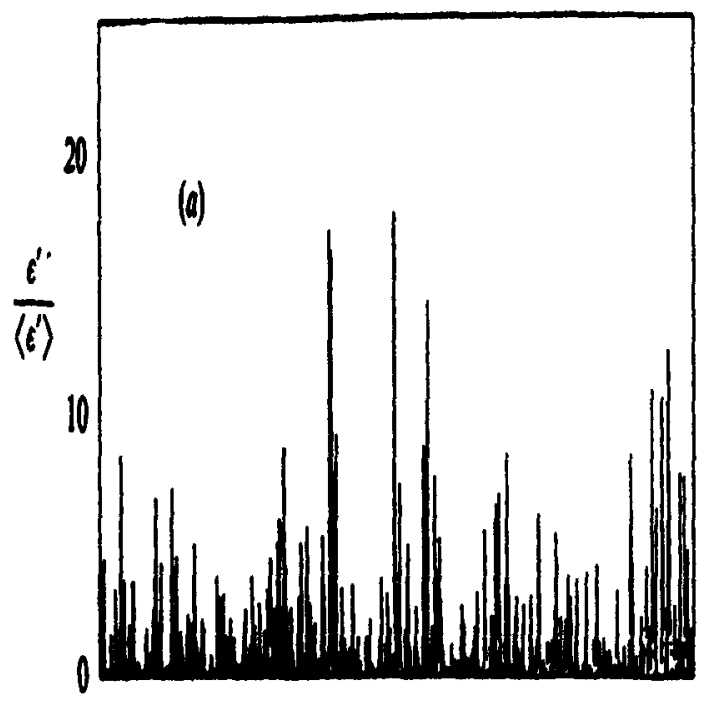

Figure 2. Typical signals of a representative component of the surrogate for the energy dissipation $\epsilon^{\prime}$ (normalized by its mean) versus time $t$ from an atmospheric boundary layer at a moderate Reynolds number (after Meneveau and Sreenivasan [50]).

$C=0.5 \pm 0.05$. However, the kurtosis $K \equiv \mathcal{S}_{4}(r) /\left(\mathcal{S}_{2}(r)\right)^{2}$, which is a measure of the flatness of the distribution of velocity differences, has values larger than the K41 prediction as $r \rightarrow 0$ [11], which indicates that the velocity gradients are intermittent (i.e., large values of velocity differences are more probable than in K41). This suggests, in turn, that the energy dissipation is nonuniform, contrary to one of the assumptions made by Kolmogorov in $\mathrm{K} 41$.

The validity of K41 can be tested experimentally by the measurement of high-order $(p \gg 3)$ structure functions, which can capture rare events. Such experiments show that

$$
\mathcal{S}_{p}(r) \sim r^{\zeta_{p}}
$$

the $\mathrm{K} 41$ prediction is $\zeta_{p}=\zeta_{p}^{K 41}=p / 3$. Even though the measurement of high-order structure functions is a difficult task, there are good data from the recent experiments [31,32,33] which show that: (1) the $\zeta_{p}$ 's are monotonically increasing, nonlinear functions of $p$ (i.e., we have multiscaling); (2) the deviation from the K41 prediction $\delta \zeta_{p} \equiv p / 3-\zeta_{p}$ is an increasing function of $p$ and starts becoming significant for $p \geq 4$ (figure 1).

We mention in passing that some experimental studies [34] indicate that $\delta \zeta_{p}$ decreases with increasing $\mathrm{Re}$, as also suggested by some theories $[35,36]$; there are also suggestions [37] of $(\ln \mathrm{Re})^{-1}$ corrections to $\mathrm{K} 41$. However, we believe it is fair to say that the general experimental position $[2,33]$ is that the multiscaling corrections to K41 persist. A careful study over a wide range of Re, possible, e.g., in flows in Helium gas [38,39], should be able to resolve this potentially significant point. Another intriguing issue has been raised by recent experiments on both open [38] and closed [39] flows in Helium gas: these provide some evidence of a transition or a crossover (in, e.g., the kurtosis $K$ ) with increasing $\mathrm{Re}$. Careful studies, already under way [40], must resolve whether these 


\section{Sujan $K$ Dhar et al}

transitions are real or arise because of the limitations of responses of the probes used in these measurements.

How might multiscaling corrections to K41 arise? Experimental data (figure 2) show that the energy dissipation rate $\epsilon$ is strongly intermittent. This is ignored in the K41 theory. High-order velocity structure functions are sensitive to the rare but large events which lead to intermittency, hence the discrepancy between K41 and the experimental $\zeta_{p}$ 's for large $p$.

Many phenomenological theories have been proposed to account for the intermittency of $\epsilon$. The oldest is the refined similarity hypothesis (RSH) of Kolmogorov [41], which replaces $\langle\epsilon\rangle$ in (2) by $\epsilon_{r}$, the energy dissipation rate averaged over a sphere of radius $r$, i.e.,

$$
\mathcal{S}_{p}(r) \sim\left\langle\epsilon_{r}^{p / 3}\right\rangle r^{p / 3}
$$

The validity of the RSH has been tested to some extent [42-45], but the issue is certainly not closed as noted by Chen et al [46] on the basis of direct numerical simulations $(\S 3$ ) and Sreenivasan and Antonia [2]. However, it is often used as a good working hypothesis. If, in the inertial range, we further assume the scaling form

$$
\left\langle\epsilon_{r}^{p}\right\rangle \sim r^{\tau_{p}}
$$

it follows from (4) and (5) that

$$
\zeta_{p}=p / 3+\tau_{p / 3}
$$

Note that the RSH yields a relation between the statistics of velocity differences and the unknown behaviour of $\left\langle\epsilon_{r}^{p / 3}\right\rangle$. An assumption about the latter yields $\tau_{p}$ and hence $\zeta_{p}$. In his 1962 theory, Kolmogorov assumed that $\epsilon_{r} /\langle\epsilon\rangle$ has a log-normal distribution, which, along with the RSH, and the constraint $\tau_{1}=0$, gives

$$
\tau_{p}^{K 62}=\mu p(1-p) / 2
$$

or

$$
\zeta_{p}^{K 62}=\frac{p}{3}+\mu \frac{p}{6}\left(1-\frac{p}{3}\right)
$$

where the intermittency exponent $\mu=-\tau_{2} \simeq 0.2$. This yields [47] better agreement with experiments (figure 1) than $\mathrm{K} 41$; however, for large $p$, deviations are noticeable and, as many authors have noted [2], there are several problems with a log-normal distribution for $\epsilon_{r} /\langle\epsilon\rangle$.

Other phenomenological theories include the $\beta$ model, proposed by Frisch, Sulem and Nelkin [48], and the multifractal description of turbulence, postulated by Parisi and Frisch [49]. The $\beta$ model yields

$$
\begin{aligned}
\zeta_{p}^{\beta \text { model }} & =p / 3+(3-D)(1-p / 3), \\
E(k)^{\beta \text { model }} & \sim k^{-5 / 3-(1-D / 3)} .
\end{aligned}
$$

Here $D \equiv \ln \beta / \ln b$, identified with the fractal dimension of the space filled by the eddies (at each stage of the cascade the linear size of the eddy is scaled down by a factor $b>1$ and $\beta$ is the ratio between the volumes of mother and daughter eddies), is used as a fitting parameter. Figure 1 shows a fit to $\zeta_{p}$ with $D=2.8$; clearly, the $\beta$-model fit overestimates 
$\zeta_{p}$ for $8 \lesssim p$. It also yields an inertial-range energy spectrum which is steeper than the $-5 / 3$ spectrum of $\mathrm{K} 41$.

It is easy to see [8] that the Navier-Stokes equation (see below) for an incompressible fluid is invariant under the scale transformation $r \rightarrow b r, v \rightarrow b^{h} v, \nu \rightarrow b^{1+h} \nu, t \rightarrow b^{1-h} t$, with an arbitrary exponent $h$. It has been suggested [49] that, at high $\operatorname{Re}$, i.e., small $\nu$, one must account for multifractality with many exponents $h$ as has been shown explicitly [50] for $\epsilon$ (see below): Global scale-invariance (as in $\mathrm{K} 41$ ) is replaced by local scale-invariant structures, i.e., the scaling, or Hölder, exponent $h$ varies at different points in the fluid, which is considered to be a superposition of fractal subsets, each with an $h$-dependent dimension $D(h)$. Structure functions, which now have contributions from each fractal subset, can be written as

$$
\mathcal{S}_{p}(r) \sim \int\left(r / L_{I}\right)^{h p+3-D(h)} \mathrm{d} h
$$

where $L_{I}$ is the integral scale. A saddle-point estimate yields (cf., (4))

$$
\zeta_{p}=\min _{h}[h p+3-D(h)]
$$

or

$$
D(h)-3=\max _{p}\left[p h-\zeta_{p}\right] .
$$

The K41 theory corresponds to a single exponent $h=1 / 3$ with $D(h)=3$.

The emergence of multifractal scaling for $\epsilon$ through an asymmetric breakdown of eddies has been discussed in detail and substantiated experimentally in the elegant studies of Meneveau and Sreenivasan [50]. In their picture, an eddy of size $\ell$ in $d$ dimensions breaks up into $2^{d}$ eddies of equal size $\ell / 2$. The key assumption in this model is that the energy flow to these smaller eddies takes place asymmetrically. The simplest nontrivial choice is that a fraction $p_{1}$ of the total energy gets distributed equally to half of these new $2^{d}$ eddies; the remaining fraction $\left(p_{2}=1-p_{1}\right)$ of energy goes to the other half. These eddies, in turn, break up into smaller eddies, and this process is repeated with fixed $p_{1}$, until one obtains eddies of the order of the dissipation scale. A one-dimensional section of the resulting multifractal set should look qualitatively similar to the energy dissipation signal shown in figure 2. Indeed, Meneveau and Sreenivasan [50] have shown that such a multiplicative process with $p_{1}=0.7$ yields a multifractal set which has the same singularity spectrum as the one obtained by a multifractal analysis of the data in figure 2.

Recently, She and Leveque (SL) [51] proposed a simple model which leads to a prediction for $\zeta_{p}$ in very good agreement with experimental results. This model is based on a set of hypotheses about the statistics of small-scale fluctuations in turbulence. These lead to a formula for $\zeta_{p}$, which has no adjustable parameters. The SL hypotheses are:

(i) The RSH (5) and (6) is assumed to hold.

(ii) Furthermore the moments of the energy dissipation are assumed to obey the hierarchy

$$
\frac{\left\langle\epsilon_{\ell}^{p+1}\right\rangle}{\epsilon_{\ell}^{(\infty)}\left\langle\epsilon_{\ell}^{p}\right\rangle}=A_{p}\left(\frac{\left\langle\epsilon_{\ell}^{p}\right\rangle}{\epsilon_{\ell}^{(\infty)}\left\langle\epsilon_{\ell}^{p-1}\right\rangle}\right)^{\beta}, \quad 0<\beta<1,
$$


where $A_{p}$ are constants and $\epsilon_{\ell}^{(\infty)} \equiv \lim _{p \rightarrow \infty}\left\langle\epsilon_{\ell}^{p+1}\right\rangle /\left\langle\epsilon_{\ell}^{p}\right\rangle$ is a quantity that is sensitive to the tail of the distribution of $\epsilon_{\ell}$, i.e., to very intermittent structures. SL postulate that the hierarchy originates from some hidden statistical symmetry of the NavierStokes equation.

(iii) The quantity $\epsilon_{\ell}^{(\infty)}$ appearing in the moment hierarchy (14) is associated with the most intermittent dissipative structures and SL suggest that it has a divergent scale dependence, namely,

$$
\epsilon_{\ell}^{(\infty)} \sim \ell^{-\Delta}, \quad \ell \rightarrow 0 .
$$

They argue further that $\epsilon_{\ell}^{(\infty)} \sim \delta E^{\infty} / t_{\ell}$, where $\delta E^{\infty} \sim \delta v_{0}^{2} \sim\langle\epsilon\rangle^{2 / 3} \ell_{0}^{2 / 3}$ is the largest amount of energy available for dissipation (i.e., the energy in the largest structures) and the scale-dependent energy-transfer time $t_{\ell} \sim\langle\epsilon\rangle^{-1 / 3} \ell^{2 / 3}$. The last asymptotic relation follows from dimensional analysis and the assumption that $t_{\ell}$ shows no anomalous scaling. Finally one obtains $\Delta=2 / 3$.

(iv) The most intermittent dissipative structures are assumed to be filamentary, i.e., of spatial dimension 1 or codimension 2 .

Equations (6) and (14) yield a difference equation for $\tau_{p}$ which, when solved with the boundary conditions that follow from the SL hypotheses, gives

$$
\tau_{p}^{\mathrm{SL}}=-\frac{2}{3} p+2\left[1-\left(\frac{2}{3}\right)^{p}\right]
$$

or, via (7),

$$
\zeta_{p}^{\mathrm{SL}}=\frac{p}{9}+2\left[1-\left(\frac{2}{3}\right)^{p}\right]
$$

The most important boundary condition follows from (14) and (15) and is $\lim _{p \rightarrow \infty}$ $\left(\tau_{p+1}-\tau_{p}\right)=-2 / 3$, i.e., $\tau_{p} \rightarrow-2 p / 3+C_{0}$ as $p \rightarrow \infty$; it is easy to see from (13) that $C_{0}$ is the codimension of the most singular structure, i.e., the one with the smallest Hölder exponent $h_{\min }$. Assumption (iv) yields $C_{0}=2$ which corresponds to $h_{\min }=1 / 9$.

The SL formula is in good agreement with the experimental values of $\zeta_{p}$ as can be seen from figure 1 . The success of this formula is indeed remarkable, since the validity of the assumptions made is not yet clear. Of the assumptions (i)-(iv), (i) is generally accepted to be a good working approximation. Direct numerical simulations indicate that highvorticity regions are filamentary $[52,53]$, which is used to motivate assumption (iv); we return to this point in $\S 3$. Assumption (iii) awaits detailed experimental and numerical verification. Recently, this hierarchy has been verified experimentally [54] to some extent using wind-tunnel data. Dubrulle [55] mentions that a similar hierarchy has been shown to exist in the GOY shell model (see below).

Benzi et al [32] have proposed a very interesting way of extending the region over which inertial-range exponents may be extracted from structure functions. The extended self-similarity (ESS), as the procedure is called, states that the real-space structure functions present an extended inertial scaling range in log-log plots of one structure function against another. From (4) it follows that

$$
\mathcal{S}_{p}(r) \sim \mathcal{S}_{q}(r)^{\zeta_{p} / \zeta_{q}}
$$

and Benzi et al [32] have shown, by using experimental data, that the inertial scaling 
region extends up to $r \simeq 5 \eta_{d}$ in ESS plots. (By contrast, plots of $\mathcal{S}_{p}(r)$ versus $r$ show inertial range scaling only up to $r \simeq 20 \eta_{d}$.) It is conventional to use $q=3$ in (18), so $\zeta_{p} / \zeta_{3} \simeq \zeta_{p}$ since $\zeta_{3}$ is very nearly equal to unity, when the flow is homogeneous and isotropic. (Indeed the Karman-Howarth relation [7] yields $\zeta_{3}=1$.) Thus ESS provides an effective method for determining $\zeta_{p}$ from data for which the extent of the inertial range is often restricted. However, the reason behind the success of ESS is still unclear (but see $[56,57]$ for some progress on this front).

Although ESS is an effective method for determining inertial-range scaling behaviour in some turbulent flows, it does not work well for all such flows, particularly in the presence of a strong shear. For such systems, Benzi et al [58] have proposed the generalized extended self similarity (GESS) ansatz, which they claim extends the scaling down to the smallest resolvable spatial separations. They begin with the dimensionless, real-space structure functions

$$
\mathcal{G}_{p}(r)=\frac{\mathcal{S}_{p}(r)}{\mathcal{S}_{3}(r)^{p / 3}} .
$$

If $\mathrm{K} 41$ were true, $\mathcal{G}_{p}(r)$ would be independent of $r$ in the inertial range. However, because of multiscaling, $\mathcal{G}_{p}(r)$ displays nontrivial scaling behaviour. Specifically, Benzi et al [58] propose that $\mathcal{G}_{p}(r)$ is self-scaling over all resolvable length scales, i.e.,

$$
\mathcal{G}_{p}(r)=\left[\mathcal{G}_{q}(r)\right]^{\rho_{p, q}}
$$

From the $r$-dependence of $\mathcal{S}_{p}$ (4) it follows that

$$
\rho_{p, q}=\frac{\zeta_{p}-p \zeta_{3} / 3}{\zeta_{q}-q \zeta_{3} / 3} .
$$

Thus, if $\log \mathcal{G}_{p}(r)$ is plotted against $\log \mathcal{G}_{q}(r)$ one should get a straight line with slope $\rho_{p, q}$ up to all resolvable length scales. This has been checked to some extent by Benzi et al [58] from numerical and experimental data for $(p, q)=(5,6)$.

Dissipation-range behaviors of structure functions have been studied less than their counterparts in the inertial range. There are some data for the energy spectrum $E(k)$. In particular, K41 suggests that

$$
E(k)=\langle\epsilon\rangle^{2 / 3} k^{-5 / 3} f\left(k / k_{\mathrm{d}}\right),
$$

where the dissipation scale $k_{\mathrm{d}}^{-1} \equiv \eta_{\mathrm{d}}$ and the scaling function $f\left(k / k_{\mathrm{d}}\right) \rightarrow C$, for small $k / k_{\mathrm{d}}$. The general form of $f\left(k \eta_{\mathrm{d}}\right)$ is not known, but the consensus from experiments $[2,13]$ and direct numerical studies [59] is that, in the far dissipation range, i.e., for large $k / k_{\mathrm{d}}$, $E(k) \sim k^{\delta} \exp \left(-c k / k_{\mathrm{d}}\right)$, a form first suggested by Kraichnan [60]. The exponent $\delta$ and the constant $c$ are not easy to determine; the best numerical studies [59] yield $\delta \simeq 3.3$ and $c \simeq 7.1$. It is also worth noting that $f\left(k / k_{\mathrm{d}}\right)$ does not decay monotonically with $k / k_{\mathrm{d}}$; rather, it increases mildly in the inertial range and then starts decreasing in the dissipation range leading to a slight pile up in the energy spectrum in the crossover regime. This has been referred to as a bottleneck phenomenon [61].

Soon after the introduction of ESS it was pointed out by Stolovitzky and Sreenivasan [22] that ESS plots cross over from their inertial-range asymptotic behaviour to another asymptotic behaviour in the far dissipation range that is characterized by a steeper power 


\section{Sujan $\mathrm{K}$ Dhar et al}

law (cf. (18)). This has not been explored experimentally in the GESS context. We will study this in detail in $\S 3$ in the context of our wave-vector-space (henceforth $k$ space) generalizations of ESS and GESS.

\subsection{Probability distributions}

The third category of measurements yield probability distributions for quantities such as the velocity, components of velocity differences (i.e., $\left.\delta \mathbf{v}_{i} \equiv\left[\mathbf{v}_{i}(\mathbf{x}+\mathbf{r})-\mathbf{v}_{i}(\mathbf{x})\right]\right)$, etc.
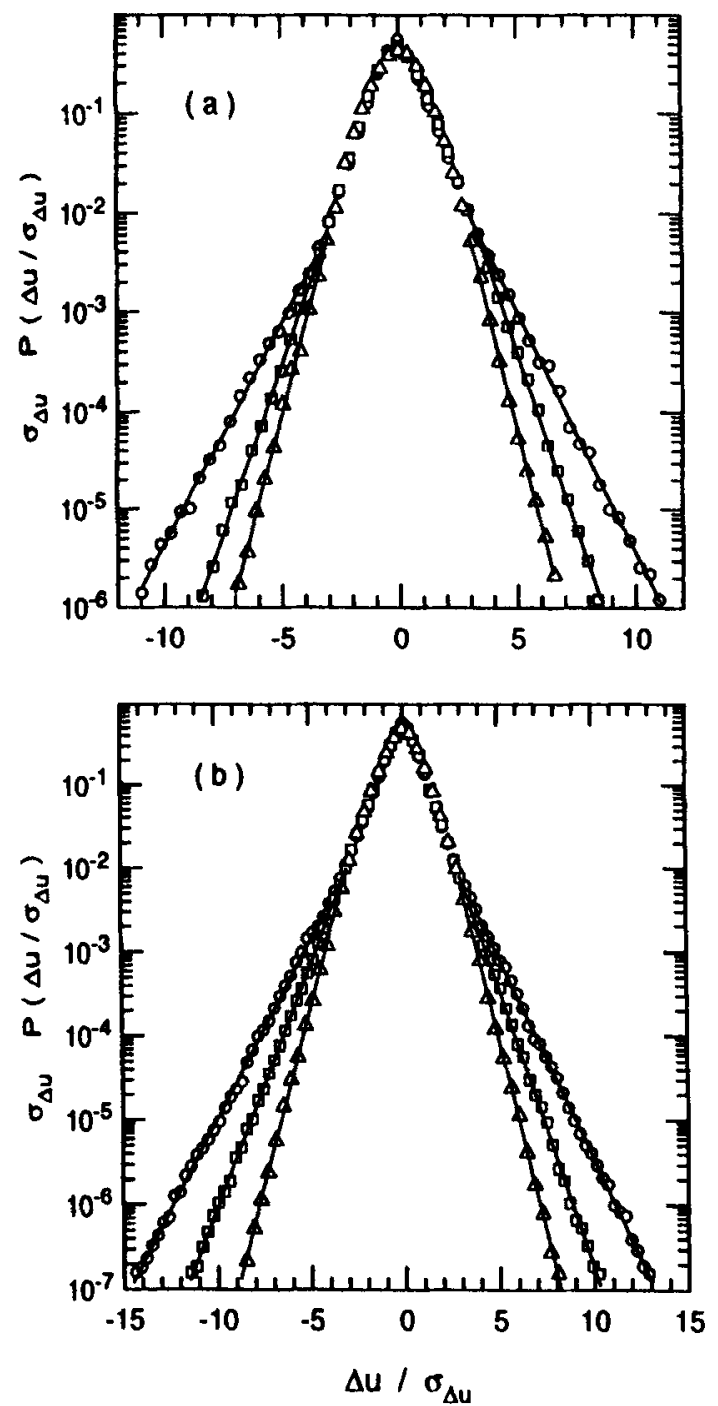

Figure 3. PDFs of velocity differences for separations $r$ within the inertial range for (a) wind-tunnel data: $r / \eta_{\mathrm{d}}=37.5(0), 351(\square)$, and $1870(\Delta)$; (b) atmospheric surface layer: $r / \eta_{\mathrm{d}}=61.3(0), 408(\square)$, and $2900(\Delta)$. The solid lines correspond to best fits which are of the exponential form (after Praskovsky and Oncley [62]). 

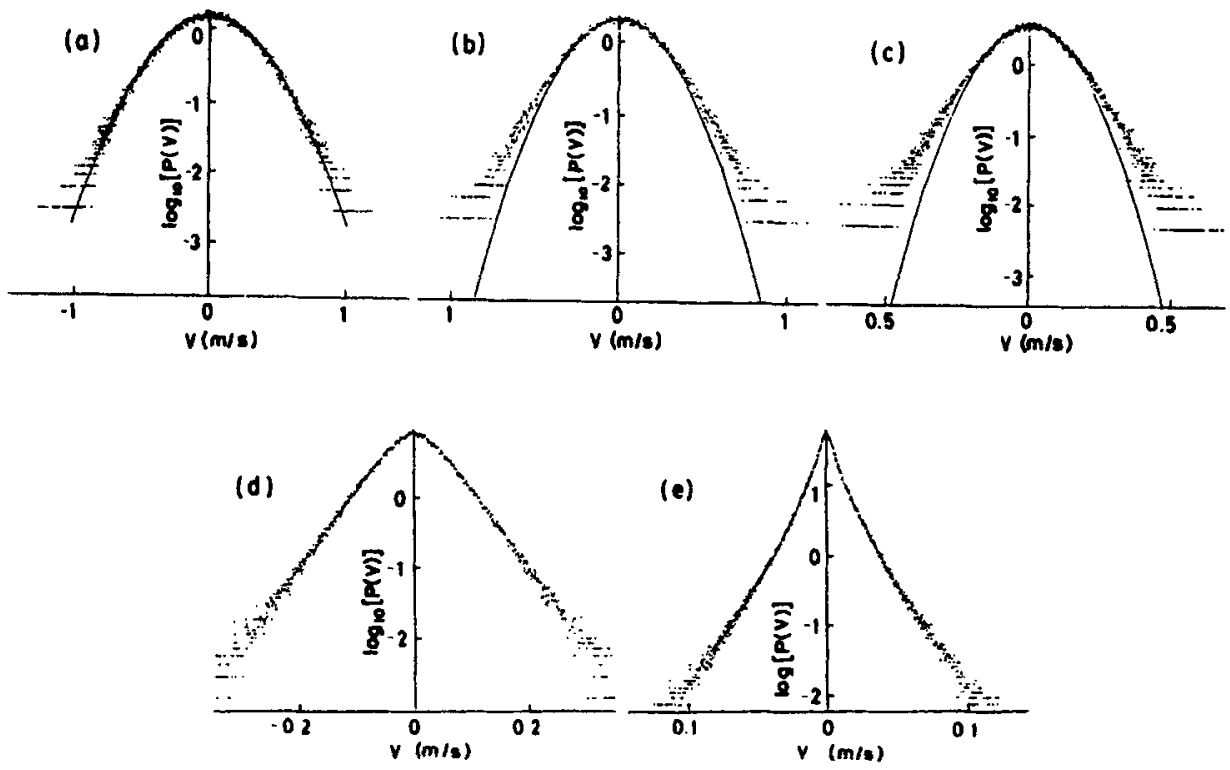

Figure 4. PDFs of band-pass-filtered signals with $f_{c}=$ (a) $0.07 \mathrm{kHz}$, (b) $0.15 \mathrm{kHz}$, (c) $0.70 \mathrm{kHz}$, (d) $3.0 \mathrm{kHz}$, and (e) $10 \mathrm{kHz}$ (see text). Solid curves show Gaussian distributions (after Katsuyama, Horiuchi and Nagata [34]).

There is considerable evidence [62] that the distribution of velocity differences is not Gaussian at small length scales in fully developed turbulence: Experimental data show that the probability distribution functions (PDFs) of velocity gradients over small distances (i.e., large wavenumbers) have a tail which falls more slowly than that of a Gaussian distribution [62,63]. It is often said [64] that such non-Gaussian behaviour is associated with the presence of small-scale coherent structures which lead to intermittency. Whereas this might well be true, it is worth noting [8] that even K41 yields a non-Gaussian, stretched-exponential distribution for the distribution of velocity gradients; however, the stretching predicted by $\mathrm{K} 41$ is less than that observed experimentally. Figure 3 shows a plot of the PDFs of velocity differences obtained by Praskovsky and Oncley [62] from wind-tunnel experiments and from the atmospheric surface layer; they fit well to an exponential form. However, a general theoretical understanding of the precise relation between small-scale coherent structures and such PDFs is still lacking.

Katsuyarna, Horiuchi and Nagata [34] have studied the behaviour of PDFs obtained from a band-pass-filtered velocity signal. In this study, the velocity time series, recorded in wind-tunnel experiments, was band-pass filtered to obtain the record $V\left(t ; f_{c}\right)$, where $f_{c}$ is the midband frequency of the filter. The PDFs of $V\left(t, f_{c}\right)$ are Gaussian at small $f_{c}$, but decay more slowly than Gaussian distributions as $f_{c}$ is increased (figure 4). A number of authors had suggested earlier $[63,65]$ that such PDFs can be approximated by stretched exponentials of the form $P(V) \propto \exp \left(-\alpha|V|^{m}\right)$. For their band-pass-filtered signals, Katsuyama et al [34] measured the stretching exponent $m$ and found that, for small $f_{c}$, corresponding to integral-range time scales, $m \simeq 2$, i.e., a Gaussian PDF; as $f_{c}$ was increased (times corresponding to inertial-range timescales), $m$ started falling below 2 .

Pramana - J. Phys., Vol. 48, No. 1, January 1997 (Part I) 


\section{Sujan $K$ Dhar et al}

This fall became more and more significant for high $f_{c}$ 's, corresponding to dissipationrange timescales.

Katsuyama et al used another method for characterising the deviation of the PDF $V\left(t ; f_{c}\right)$ from a Gaussian PDF: They calculated the normalized moments

$$
\gamma_{2 p}=\frac{(2 p-1) ! !}{2} \frac{\left\langle\left[V\left(t ; f_{c}\right)\right]^{2}\right\rangle^{p}}{\left\langle\left[V\left(t ; f_{c}\right)\right]^{2 p}\right\rangle}, \quad p=2,3,4, \ldots
$$

where $(2 p-1) ! ! \equiv(2 p-1)(2 p-3) \cdots 3.1$. For a Gaussian distribution, $\gamma_{2 p}=0.5$ for all $p$. Even a very weak deviation from a Gaussian distribution becomes apparent if we calculate $\gamma_{2 p}$ for sufficiently large $p$. The band-pass-filtered data show that $\gamma_{2 p}$ goes slightly below 0.5 for $f_{c}$ 's corresponding to inertial-range timescales. This is followed by a more rapid decay in dissipation-range timescales and, as anticipated, the deviation from Gaussian behaviour is clearly more prominent for large $p$.

Recall that, in his 1962 theory [42], Kolmogorov assumed that $\epsilon_{r} /\langle\epsilon\rangle$ has a log-normal distribution. This is not substantiated by experiments, which yield data that can be fit [50] to a stretched-exponential form with a stretching exponent of $1 / 2$; however, this latter form violates the Carleman criterion, i.e., such a PDF is not determined uniquely by its moments $[2,66]$. It has also been shown recently $[55,67]$ that the SL assumptions lead to distributions in the log-Poisson class. We are not aware of a detailed experimental verification of this.

\section{Deterministic models for the study of homogeneous isotropic turbulence}

The tasks before a theory of homogeneous, isotropic turbulence are many. Given the perspective adopted in this paper, these must include an understanding of the multiscaling behaviours of the structure functions and probability distributions defined in $\S 2$. It is also important to elucidate the role, if any, of coherent structures in determining these properties. At the moment we are very far from this goal. However, progress has been made over the past decade by using: (a) approximate analytical methods to study stochastic models, which are discussed in the companion paper by Bhattacharjee [20]; and (b) direct numerical studies of deterministic models. We concentrate on the latter in this section, which is organised as follows: It begins with an introduction to direct numerical solutions of the Navier-Stokes equation and the GOY shell model, which are used in studies of homogeneous, isotropic turbulence. This is followed by a brief overview of what has been learnt about intermittent structures, the scaling of structure functions (including ESS and GESS), and various PDFs from such studies. Our emphasis is on showing how studies of both the Navier-Stokes equation and reduced models like the GOY shell model can augment each other as we have shown in recent studies [89] of $k$-space ESS and GESS.

\subsection{Models}

The model of choice for the study of fluid turbulence is, of course, the deterministically forced Navier-Stokes (NS) equation for an incompressible fluid. If we restrict ourselves to low-Mach-number flows and cases where the equation for the energy density can be 
Table 1. Parameters $\nu$ (viscosity), $\nu_{\mathrm{H}}$ (hyperviscosity), $\operatorname{Re}_{\lambda}$ (Taylor-microscale Reynolds number), $\tau_{\mathrm{e}}$ (box-size eddy-turnover time), $\tau_{\mathrm{av}}$ (averaging time), $\tau_{\mathrm{t}}$ (transient time) and $k_{\mathrm{d}}$ (dissipation-scale wavenumber) for our $3 d$ NS runs NS1-4 ( $L=\pi, k_{\max }=64$ ) and GOY-model runs G1-8 $\left(k_{\max }=2^{22} k_{0}\right)$. The step size ( $\left.\delta t\right)$ used is 0.02 for NS1-4, $10^{-4}$ for G1-4, and $2.10^{-5}$ for G5-8. In our NS runs we estimate $k_{\mathrm{d}}=\eta_{\mathrm{d}}^{-1}$ by solving the nonlinear equation, obtained by balancing dissipative and convective time scales, $\nu \eta_{\mathrm{d}}^{-2}+\nu_{h} \eta_{\mathrm{d}}^{-4}=\langle\epsilon\rangle^{1 / 3} \eta_{\mathrm{d}}^{-2 / 3}$, which reduces to the formula given above for $\eta_{\mathrm{d}}$ if the hyperviscosity $\nu_{h}=0$.

\begin{tabular}{llllllll}
\hline Run & $\nu$ & $\nu_{\mathrm{H}}$ & $\operatorname{Re}_{\lambda}$ & $\tau_{\mathrm{e}} / \delta t$ & $\tau_{\mathrm{t}} / \tau_{\mathrm{e}}$ & $\tau_{\mathrm{av}} / \tau_{\mathrm{e}}$ & $k_{\max } / k_{\mathrm{d}}$ \\
\hline NS1 & $5.10^{-4}$ & 0 & $\simeq 3.5$ & $\simeq 3.10^{4}$ & $\simeq 1$ & 2 & $\simeq 4$ \\
NS2 & $2.10^{-4}$ & 0 & $\simeq 8$ & $\simeq 3.10^{4}$ & $\simeq 1$ & $\simeq 2.5$ & $\simeq 2.3$ \\
NS3 & $5.10^{-4}$ & $5.10^{-6}$ & $\simeq 3.5$ & $\simeq 3.10^{4}$ & $\simeq 1$ & $\simeq 1$ & $\simeq 6.5$ \\
NS4 & $5.10^{-4}$ & $10^{-6}$ & $\simeq 22$ & $\simeq 3.10^{3}$ & $\simeq 10$ & $\simeq 7$ & $\simeq 2$ \\
G1-4 & $5.10^{-6}-10^{-7}$ & 0 & $4.10^{4}-3.10^{5}$ & $\simeq(1.5-2.0) 10^{4}$ & $\simeq 500$ & $\simeq 2500 \simeq 2^{5}-2^{3}$ \\
G5-8 & $5.10^{-8}-10^{-9}$ & 0 & $3.5 .10^{5}-3.10^{6}$ & $\simeq(0.7-1) 10^{5}$ & $\simeq 500$ & $\simeq 2500 \simeq 2^{3}-1$ \\
\hline
\end{tabular}

neglected, the NS equation is

$$
\partial_{t} \mathbf{v}+(\mathbf{v} \cdot \nabla) \mathbf{v}=\nu \nabla^{2} \mathbf{v}-\nabla P / \rho+\mathbf{f},
$$

where $\mathrm{v}$ is the velocity field, $P$ the pressure, $\rho$ the density, $\nu$ the kinematic viscosity, $\mathrm{f}$ the external, deterministic force, and incompressibility is enforced by the condition

$$
\nabla \cdot \mathbf{v}=0
$$

Equations (24) and (25) must be solved with suitable boundary conditions and initial conditions; periodic boundary conditions are convenient in studies of homogeneous, isotropic turbulence. At high $\mathrm{Re}$, little can be said analytically about the statistical steady state of this driven equation beyond the phenomenological theories described in $\S 2$ or by using stochastic models [20]; one must, perforce, obtain information about this steady state from direct numerical solutions (DNS). In studying the properties of homogeneous and isotropic turbulence, it is sometimes convenient to work in $k$ space; here the equation is,

$$
\partial_{i} v_{i}(\mathbf{p})+i M_{i j k}(\mathbf{p}) \sum_{\mathbf{q}} v_{j}(\mathbf{q}) v_{k}(\mathbf{p}-\mathbf{q})=-\nu p^{2} v_{i}(\mathbf{p})+f_{i}(\mathbf{p})
$$

with $\mathbf{p}$ and $\mathbf{q}$ denoting wave vectors, $M_{i j k}(\mathbf{p}) \equiv\left[p_{j} P_{i k}(\mathbf{p})+p_{k} P_{i j}(\mathbf{p})\right] / 2, P_{i j}(\mathbf{p}) \equiv$ $\left[\delta_{i j}-p_{i} p_{j} / p^{2}\right]$ the transverse projector, and Fourier transforms are implied by wavevector arguments. Typically one uses periodic boundary conditions and, if a hyperviscosity $\nu_{H}$ (table 1) is used, $\nu p^{2}$ in (26) is replaced by $\left(\nu+\nu_{H} p^{2}\right) p^{2}$. In numerical studies it is advantageous to use a pseudo-spectral scheme that evaluates certain terms in real space and others in $k$ space. Such studies began with the pioneering work of Orszag and Patterson [68]. Subsequent studies, carried out by many groups, include refs. [28,52,53,59,69-76]. We follow Meneguzzi and Vincent [73] for our NS simulation.

In spite of rapid advances in computing power, direct numerical studies of the NS equation remain daunting at high $\mathrm{Re}$. The largest studies have used $512^{3}$ Fourier modes for the velocity field and achieved a Taylor-microscale Reynolds number 
$\operatorname{Re}_{\lambda} \equiv v_{\mathrm{mns}} \lambda / \nu \simeq 220 ;$ here the Taylor-microscale is [73] $\lambda \equiv\left[\int E(k) \mathrm{d} k / \int k^{2} E(k) \mathrm{d} k\right]^{1 / 2}$ and $v_{\mathrm{mms}} \equiv\left[2 \int_{0}^{\infty} E(k) \mathrm{d} k\right]^{1 / 2}$ is the root-mean-square velocity. Larger studies are not feasible yet because computational costs scale as $\operatorname{Re}^{9 / 4}$, for storage requirements, and $\mathrm{Re}^{1 / 2}$ for the number of arithmetic operations. Important time scales include the integral and box-size eddy-turnover times defined, respectively, as $\tau_{I} \equiv L_{I} / v_{\text {rms }}$ and $\tau_{e} \equiv L / v_{\text {rms }}$, where $L_{I} \equiv\left[\int k^{-1} E(k) \mathrm{d} k / \int E(k) \mathrm{d} k\right]^{1 / 2}$ is the integral scale and $L$ is the box size . For our four runs for the NS equation (table 1) $\tau_{e} \simeq 8 \tau_{I}$; we report all times in terms of the former.

Given these computational constraints it is useful to work with simplified models that are physically motivated and capture as many aspects of fluid turbulence as possible. Though such models must, perforce, make several approximations (see below), they can allow us to explore the large-Re regime, which is crucial for the resolution of many questions about the asymptotic behaviours of structure functions, etc. The most successful models of this type are shell models. Of these the one due to Gledzer [77] and Ohkitani and Yamada [78], infelicitously called the GOY model, has been studied most over the past five years; and, as we show below, the GOY model yields, in spite of its simplicity, results that are not only physically instructive but also in remarkably good agreement with experiments.

The Richardson-cascade picture suggests that the scaling behaviour seen in fluid turbulence might well arise in a simplified dynamical system with a reduced number of degrees of freedom arranged hierarchically. This key idea motivated the seminal studies of Obukhov [79], Gledzer [77], and Desnyansky and Novikov [80], who postulated shell models quite similar to, though not as succesful as, the GOY model, on which we concentrate here. Other more complicated shell models are mentioned briefly below; we refer the reader to ref. [8] for a more extensive coverage of these.

Such shell models must have the symmetries of the NS equation. Unfortunately, it is not clear whether we know all the relevant symmetries; but one can at least ensure the essential conservation laws (of energy and helicity) hold in the inviscid and unforced limit. Furthermore, to attain much larger Reynolds numbers than is possible in direct numerical simulations of the NS equation, the "shells" in $k$ space are logarithmically spaced. In the GOY model they are the one-dimensional set of wave vectors $k_{n}$, labelled by shell numbers $n$ :

$$
k_{n}=k_{0} \lambda^{n}, \quad n=1,2, \ldots, N,
$$

where $\lambda$ is the ratio between wave vectors in nearest-neighbour shells, $k_{0}$ sets the scale of wave vectors, and $N$ is the total number of shells. The dynamical variables are the complex scalar velocities $v_{n}$ associated with the shells; these can be considered to represent the Fourier transform of the velocity characterising an eddy of scale $\sim k_{n}^{-1}$. The evolution equation for $v_{n}$ is

$$
\frac{\mathrm{d}}{\mathrm{d} t} v_{n}+\nu k_{n}^{2} v_{n}=F_{n}+i C_{n}
$$

where $\nu$ is the kinematic viscosity, $F_{n}$ is a forcing term, and $C_{n}$ is the nonlinear coupling between different shells. Thus the NS equation is approximated by a dynamical system comprising $2 N$ ordinary differential equations (each $v_{n}$ has both real and imaginary parts). Rigid boundary conditions are imposed on the $v_{n}$ 's, i.e., $v_{n}=0$ for $n<1$ and

Pramana - J. Phys., Vol. 48, No. 1, January 1997 (Part I) 
$n>N$. The forcing term has a constant amplitude and is typically chosen to act on a particular shell $n^{\prime}$, i.e.,

$$
F_{n}=F_{0} \delta_{n, n^{\prime}}
$$

with $n^{\prime}$ the index of the shell on which the force acts. As described in earlier studies [78,81-85], the usual choice is $n^{\prime}=4$, but Kadanoff, Lohse and Schörghofer [86] note that the choice $n^{\prime}=1$ (which we adopt in our dynamical studies below) does not change the behaviour of the model in the static case. To mimic the advection terms in the NS equation, the nonlinear term $C_{n}$ in (28) should have terms like $k_{n} v_{n^{\prime}} v_{n^{\prime \prime}}$; but, since the Richardson-cascade picture suggests that an eddy of a particular length scale interacts significantly only with eddies of nearby scales, such interaction terms are chosen to be local in $k$ space (i.e., $n^{\prime}$ and $n^{\prime \prime}$ are chosen to be close to $n$ ). The GOY form of $C_{n}$ is

$$
C_{n}=a_{n} k_{n} v_{n+1}^{*} v_{n+2}^{*}+b_{n} k_{n-1} v_{n-1}^{*} v_{n+1}^{*}+c_{n} k_{n-2} v_{n-1}^{*} v_{n-2}^{*},
$$

with $a_{N-1}=a_{N}=b_{1}=b_{N}=c_{1}=c_{2}=0$; thus the velocity of a shell is affected only by the velocities of its nearest- and next-nearest-neighbour shells. To specify $C_{n}$ completely we must fix the constants $a_{n}, b_{n}, c_{n}$. The first constraint is that the kinetic energy $E=\sum_{n}\left|v_{n}\right|^{2} / 2$ must be conserved in the inviscid $(\nu=0)$ and unforced $\left(F_{n}=0\right.$ for all $n$ ) limit. This yields, $a_{n}+b_{n+1}+c_{n+2}=0$. These coefficients are defined modulo a multiplicative factor, so that one can fix $a_{n}=1$ without loss of generality, whence

$$
a_{n}=1 ; \quad b_{n}=-\delta ; \quad c_{n}=-1+\delta .
$$

The parameter $\delta$ does not have an analogue in NS dynamics, but it plays an important role in the GOY shell model since it determines the ratio between the backward (towards smaller $k$ ) and forward (towards larger $k$ ) energy cascades as shown by Biferale, Lambert, Lima, and Paladin [87]. The choice $\lambda=(1-\delta)^{-1}$ (in most studies $\delta=1 / 2$ and $\lambda=2$ ), in addition to conserving the kinetic energy, also conserves $H=\sum(-)^{n} k_{n}\left|v_{n}\right|^{2}$ in the inviscid and unforced limit [88]. Kadanoff, Lohse, Wang and Benzi [84] have noted that $H$ is the GOY-model analogue of the helicity, which is conserved in the inviscid, unforced NS equation. Note also that the term $C_{n}$ in the GOY model conserves the phase-space volume, i.e., the total volume in the $2 N$-dimensional velocity space, since $\partial C_{n} / \partial v_{n}=0$. And, as in the NS equation, the GOY model is forced near small $k$, but the dissipation occurs principally at large $k$ 's, which, along with the nonlinear coupling, ensures a cascade of energy from small to large wavenumbers. Of course the GOY shell model is not derivable from the NS equation in any obvious way. But its simplicity and numerical tractability have inspired quite a few studies, principally numerical simulations, some of which we discuss below. Some limitations of the GOY shell model are discussed towards the end of this section.

It is easy to see that the Kolmogorov scaling relation $v_{n} \sim k_{n}^{-1 / 3}$ for the GOY model is a solution of the static problem in the inviscid and unforced limit. It has been shown [87] that static solutions of the GOY model, in the inviscid, unforced limit, are the fixed points of the map $q_{n}=\delta / 2+(1-\delta) / 4 q_{n-1}$, where $q_{n} \equiv v_{n+3} / v_{n}$. The K41-type solution, $v_{n} \sim k_{n}^{-1 / 3}$, corresponds to the fixed point $q=1 / 2$. For the dynamic but unforced and inviscid case, this $\mathrm{K} 41$ fixed point becomes unstable (for $\delta=1 / 2$, see below), but continues to play a crucial role for the dynamics with finite viscosity and forcing. Before embarking on a dynamical study of the GOY model, it is, therefore, useful to understand

Pramana - J. Phys., Vol. 48, No. 1, January 1997 (Part I) 


\section{Sujan $K$ Dhar et al}

the nature of its static solutions. We refer the reader to refs $[87,92]$ for detailed discussions of such solutions.

Though the GOY-model equations look simple, they cannot be solved analytically. Thus one must resort to direct numerical studies, which must be carried out carefully since the equations form a stiff system. We follow the numerical scheme of ref. [83].

In addition to the choice of the constants $a, b, c$ and $\lambda$ given in eq. (31), we must specify the forcing term (29). We choose: $F_{0}=5(1+i) 10^{-3}, n^{\prime}=1$, i.e., we force the first shell, not the fourth as in most earlier studies [81-84]. However, we have checked explicitly that the scaling behaviour of the model is the same with $n^{\prime}=4$ and $n^{\prime}=1$. The advantage of using $n^{\prime}=1$ is that the inertial-range is broader, so we obtain better estimates of the inertial-range exponents. Furthermore, we set $k_{0}=2^{-4}$ and work with $N=22$. Since we set $\lambda=2$, this leads to a wavenumber range of $2^{-3} \leq k_{n} \leq 2^{18}$. Earlier studies [82-84] have shown that the choice $N=22$ leads to a broad wavenumber regime, which, depending on $\mathrm{Re}$, yields sizeable inertial and dissipation ranges. Data for our GOY-model runs are given in table 1.

Biferale and Kerr [90] have pointed out that the GOY shell model has an apparent asymmetry between the odd and even shells. This does not have any counterpart in NS dynamics. To overcome this they have proposed two velocity fields per shell that transport positive- and negative-helicity components, respectively. Studies of such models are in their infancy and we refer the reader to ref. [90] for details. Even more realistic are the hierarchical shell models studied by Eggers and Grossmann [95] and Grossmann and Lohse [96]. Their scheme, also referred to as the reduced wavevector approximation (REWA), will be described briefly below.

We are now in a position to discuss some of the results that have been obtained by direct numerical studies of the NS equation and the GOY model. We begin with visualisations of intermittent structures, then discuss structure functions, ESS, and GESS, and end with probability distributions.

\subsection{The visualisation of intermittent structures}

Siggia [52] was the first to provide numerical evidence that, at large Re, regions of large $|\omega|$ (where the vorticity $\omega \equiv \nabla \times \mathbf{v}$ ) are fairly long-lived, tube-like structures. The existence of these filamentary, high-vorticity tubes has been confirmed by subsequent high-resolution runs $[53,69,71,73]$. As the value of $|\omega|$ in an iso- $|\omega|$ plot is decreased, the vorticity tubes thicken until they eventually become sheet-like. This is illustrated in figure 5, which has been obtained from our relatively low-resolution pseudo-spectral simulation that uses $64^{3}$ Fourier modes. In figure 5 we superimpose the velocity field (white arrows) on the iso- $|\omega|$ surfaces and in their vicinities; the circulation of the velocity vectors is as expected around regions of high vorticity. She et al [53] have noted that the velocity field around such vortex tubes exhibits swirling motion with a sizeable component along the local vortex-tube axis, which might lead to a local weakening of nonlinear effects $[2,93]$. The viewing angles in these photographs have been chosen to display the tubular nature of the filaments clearly.

The existence of such filamentary structures is a crucial input into the She-Leveque model $(\S 2)$. Since this model relies on a set of assumptions for the small-scale behaviour of the energy dissipation $\epsilon$, it is interesting to check whether the existence of vorticity

$$
\text { Pramana - J. Phys., Vol. 48, No. 1, January } 1997 \text { (Part I) }
$$



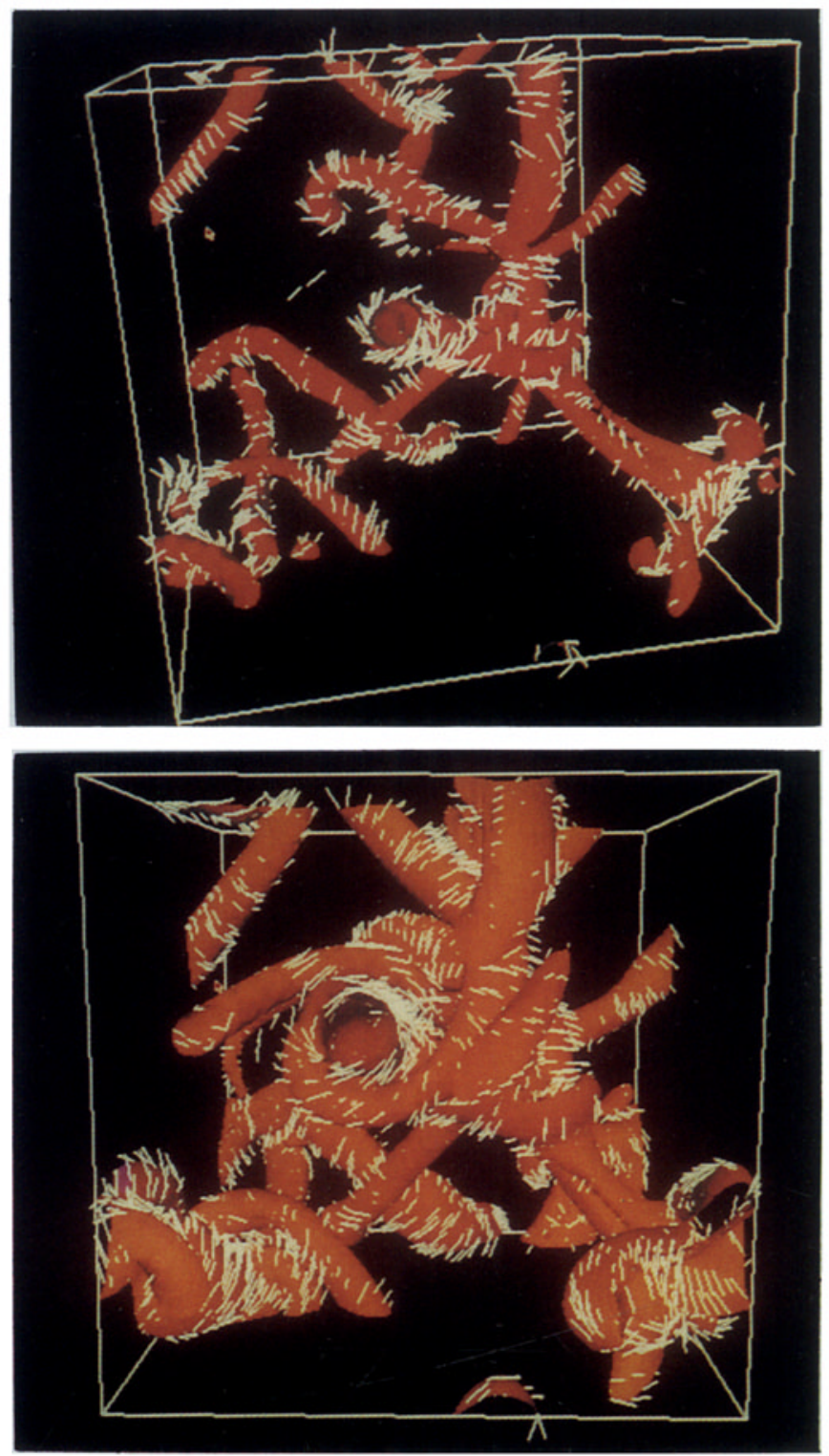

Figure 5. Iso- $|\omega|$ surfaces with superimposed velocity vectors (at points near these surfaces) obtained from an instantaneous snapshot of the velocity and vorticity fields from our run NS4 (table 1). The iso- $|\omega|$ surfaces go through points with fixed $|\omega| /|\omega|_{\max }=\mathcal{O}$, where $|\omega|_{\max }$ is the maximum value of $|\omega| ;$ the velocity vectors are shown by white arrows whose lengths are proportional to their magnitudes and whose heads are too small to be seen. (The small red arrowhead is a pointer that should be ignored here and in all subsequent photographs.) The red surface (top plate) goes through points with $\mathcal{O} \simeq 0.31$ and the orange surface (bottom plate) goes through points with $\mathcal{O} \simeq 0.12$; this illustrates the thickening of the vorticity filaments with decreasing $|\omega|$. These are perspective views with the simulation box shown by a white boundary; we use periodic boundary conditions.

Pramana - J. Phys., Vol. 48, No. 1, January 1997 (Part I) Special issue on "Nonlinearity \& Chaos in the Physical Sciences" 

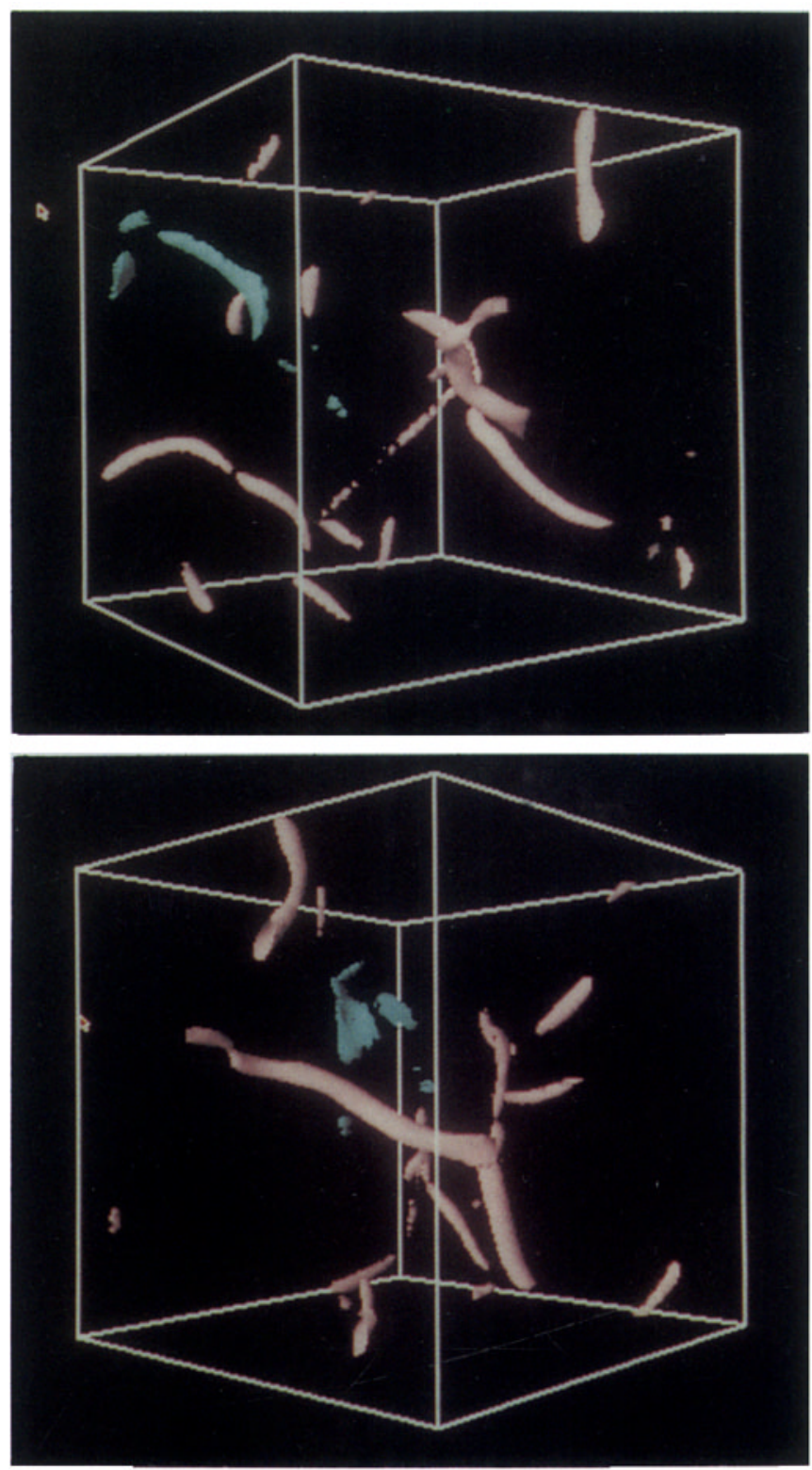

Figure 6. Superimposed iso- $|\omega|$ and iso- $\epsilon$ surfaces obtained from an instantaneous snapshot of the vorticity and dissipation fields from our run NS4 (table 1). The creamcoloured surfaces go through points with fixed $|\omega| /|\omega|_{\max }=\mathcal{O}$ whereas blue-green surfaces go through points with fi $x_{-} d \epsilon / \epsilon_{\max }=\mathcal{E}$ (the subscript $\max$ denotes maximum value). Here $\mathcal{O}=\mathcal{E}=0.5$. The top figure (a) is a rotated version of the bottom one (b). The iso- $|\omega|$ surfaces clearly show that high-vorticity regions are filamentary; however, the iso- $\epsilon$ surfaces seem more like shredded sheets or blobs. Also, high-vorticity and high-dissipation regions do not coincide. These are perspective views with the simulation box shown by a white boundary; we use periodic boundary conditions.

Pramana - J. Phys., Vol. 48, No. 1, January 1997 (Part I) 

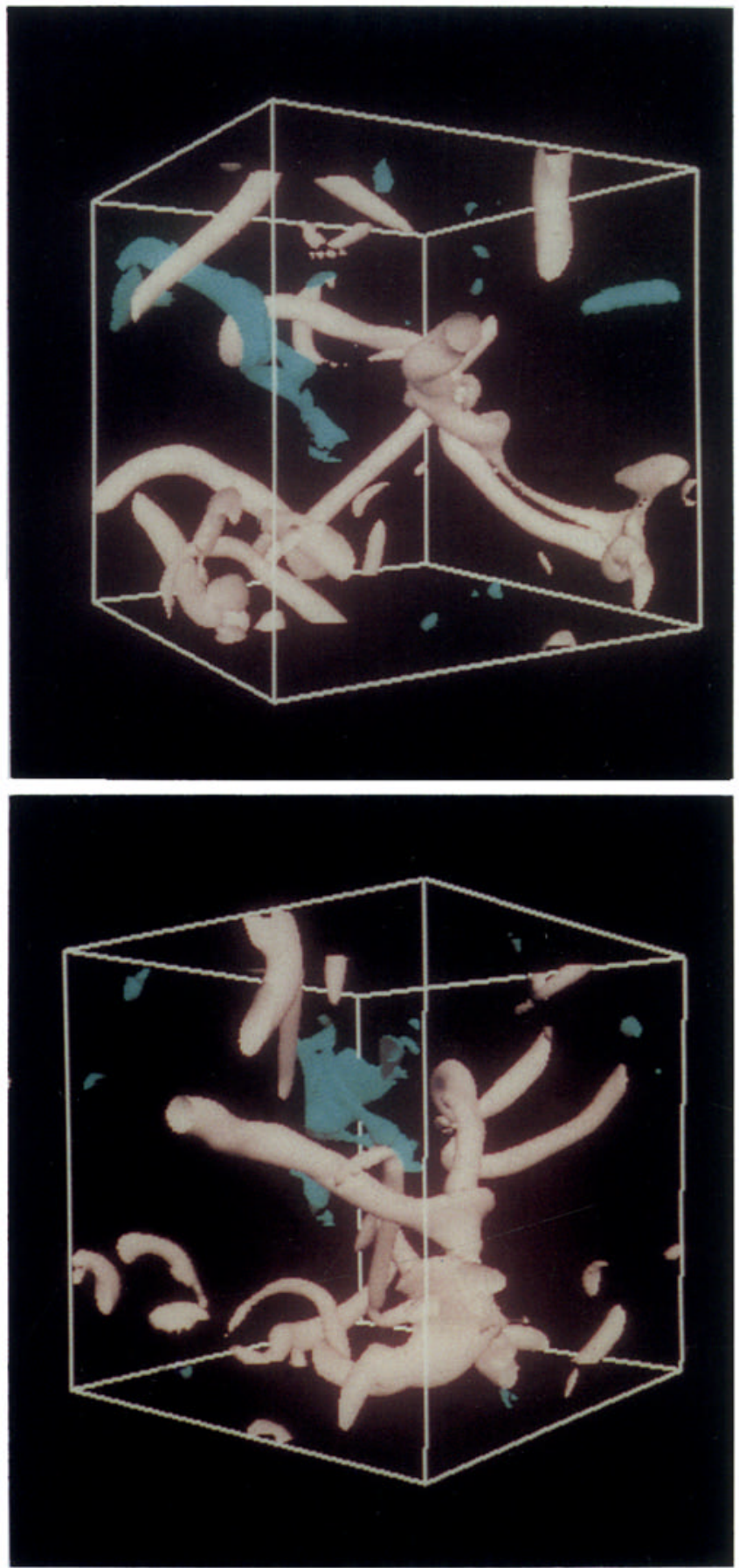

Figure 7. The same as figure 6 but with $\mathcal{O}=\mathcal{E} \simeq 0.33$, so the vorticity tubes are thicker; however, the iso- $\epsilon$ surfaces still seem like shredded sheets or blobs. The top figure (a) is a rotated version of the bottom one (b).

Pramana - J. Phys., Vol. 48, No. 1, January 1997 (Part I) 
tubes is associated with filamentary regions of large energy dissipation. (Though the averages of $\epsilon$ and $\nu \omega^{2}$ over the full box volume are the same, their spatial distributions might well be different.) Indeed, even the early numerical studies of Siggia [52] and the study of the Taylor-Green vortex by Brachet et al [94] had indicated that regions of high vorticity are regions of low $\epsilon$. This is illustrated in figures 6 and 7 , which we have obtained from an instantaneous snapshot of the real-space velocity field in our numerical study of the NS equation (run NS4 in table 1); the $\omega$ and $\epsilon$ fields follow from this velocity field. In figures 6 and 7 the cream-coloured iso- $|\omega|$ surfaces show well-formed tubes for the $|\omega|$ values used. The blue-green iso-dissipation surfaces show clearly that regions of large $\epsilon$ are, by contrast, like small shredded sheets or blobs (cf. [52]), which have no overlap with the high-vorticity tubes. Nor does their codimension seem to be the same as that of the vortex filaments. (Cream-coloured surfaces go through points with fixed $|\omega| /|\omega|_{\max }=\mathcal{O}$ whereas blue-green surfaces go through points with fixed $\epsilon / \epsilon_{\max }=\mathcal{E}$; and, to make the comparison of these surfaces as meaningful as possible, we use equal ratios, i.e., $\mathcal{O}=\mathcal{E}$.) The precise implications of this for the derivation of the SL formula $(\S 2)$ are unclear. If its success in predicting $\zeta_{p}$ is any clue, there might be a way of deriving it without using the precise codimensions of high-dissipation, as opposed to high-vorticity, regions.

It is debatable whether any real-space structures can be obtained reliably from the GOY model, which is defined on a logarithmically discretised $k$ space. Some authors have recently proposed [8] a procedure for generating an artificial velocity field $\mathbf{v}(\mathbf{r}, t)$ in three-dimensional real space from the shell-model velocity fields. The first step is to introduce a three-dimensional wavevector $\mathrm{k}=k_{n} \mathrm{e}_{n}$, where $k_{n}$ is the wavenumber of the $n$-th shell and the $e_{n}$ 's vectors of unit norm. Next the $j$-th component $(j=x, y, z)$ of the real-space velocity field is constructed through some kind of an "inverse Fourier transform"

$$
v_{j}(\mathbf{r}, t)=\sum_{n=1}^{N} C_{n}^{(j)}\left[v_{n}(t) e^{i \mathbf{k}_{n} \cdot \mathbf{r}}+\text { c.c. }\right]
$$

where the coefficients $C_{n}^{(j)}$ are random numbers of $O(1)$ and c.c. denotes complex conjugate. It can be checked that the resulting $v_{j}$ 's obey the incompressibility condition (25), since the coefficients satisfy $\sum_{j=1}^{3} C_{n}^{(j)} e_{n}^{(j)}=0$, for all $n$. However, it remains to be checked explicitly whether the velocity fields calculated by using such a prescription conform with those seen experimentally.

\subsection{Structure functions, ESS and GESS}

The scaling of real-space structure functions $\mathcal{S}_{p}(r)$ has been studied via numerical simulations of the NS equation by several grcups $[71,73,76]$. Agreement with K41 is good for $p \leq 3$ and $E(k)$ follows the $-5 / 3$ law well [73]. Some numerical studies have found that the RSH (5) holds reasonably well. However, the most recent study has cautioned that the result depends on which surrogate (see $\S 1$ ) of $\epsilon$ is used. Specifically, if one defines $\epsilon^{i j} \equiv \nu\left(\partial \mathbf{v}_{i} / \partial \mathbf{x}_{j}\right)^{2}$, then the RSH holds better with the surrogate $\epsilon^{11}$ than with $\epsilon^{21}$, in agreement with recent experiments.

Pramana - J. Phys., Vol. 48, No. 1, January 1997 (Part I) 
As regards multiscaling, data for $\zeta_{p}$ from direct numerical studies of the NS equation $[71,73]$ show significant deviations from K41 for $p>3$; however, it is still not feasible computationally to attain very large $\operatorname{Re}_{\lambda}$ (the largest so far [76] seems to be $\operatorname{Re}_{\lambda}=216$ ). Unfortunately this is not large enough to settle certain issues of principle: For example, does $\delta \zeta_{p}=p / 3-\zeta_{p} \rightarrow 0$ as $\operatorname{Re} \rightarrow \infty$, as suggested by some studies [34-36]? This is best done by using simpler models like the GOY shell model described above; of course, one must then contend with the issue of the relation of shell-model and NS results, which we discuss later.

The velocity structure functions for the GOY shell model are

$$
S_{p}\left(k_{n}\right) \equiv\left\langle\left|v_{n}\right|^{p}\right\rangle
$$

Since the $k_{n}$ 's in the GOY shell model are logarithmically spaced, the GOY-model analogue of the energy spectrum is $E(k)=\left|v_{n}\right|^{2} / k_{n}$ and the exponents $\zeta_{p}$ are given by

$$
S_{p}\left(k_{n}\right) \sim k_{n}^{-\zeta_{p}} .
$$

It is natural in the GOY model to use these $k$-space structure functions. Since we want to use direct numerical simulations of the NS equation and the GOY model in complementary ways, it is useful to study $k$-space structure functions in the NS context. We define these as

$$
S_{p}(k) \equiv\left\langle|\mathbf{v}(\mathbf{k})|^{p}\right\rangle \text {. }
$$

We use the convention that calligraphic symbols like $\mathcal{S}_{p}$ denote real-space structure functions and Roman symbols like $S_{p}$ their $k$-space analogues (not Fourier transforms). In a recent study we have provided numerical evidence that yields [89]

$$
S_{p}(k) \sim k^{-\left(\zeta_{p}+3 p / 2\right)},
$$

for $k$ in the inertial range. This difference between GOY-model and NS $k$-space structure functions arises because of phase space reasons (i.e., the logarithmic discretisation of $k$ space in the former and the usual discretisation in the latter). To have a meaningful comparison between these two models, we also define GOY-model analogues of the Taylor-microscale and the root-mean-square velocity (see above for the $3 d \mathrm{NS}$ equation): $\lambda=2 \pi / k_{1}\left[\left(\sum_{n}\left|v_{n}\right|^{2}\right) /\left(\sum_{n} k_{n}^{2}\left|v_{n}\right|^{2}\right)\right]^{1 / 2}$ and $v_{\text {rms }}=\left[\left(2 \sum_{n}\left|v_{n}\right|^{2}\right) /\left(2 \pi / k_{1}\right)\right]^{1 / 2}$, which can be used to obtain the Taylor-microscale Reynolds number $\operatorname{Re}_{\lambda}=v_{\text {rms }} \lambda / \nu$; furthermore, we use (cf., [83]) $E(k)=S_{n, 2} / k_{n}$. The time-scale associated with the smallest wavenumber is $\sim k_{1}^{-1} /\left|v_{1}\right|$; when averaged over the steady state it gives the large-scale eddy turnover time $\tau_{\mathrm{e}}$, which is the analogue of the box-size eddy-turnover time in the NS case. For our GOY-model runs we find $\operatorname{Re}_{\lambda} \sim \nu^{-0.5}$, as expected [102] at large $\nu^{-1}$. We will return to a comparison of these two models when we discuss our $k$-space versions of ESS and GESS [89] in inertial and dissipation ranges below.

Our GOY-model runs span the range $10^{4} \lesssim \operatorname{Re}_{\lambda} \lesssim 3 \times 10^{6}$. In figure 8 we show the energy spectrum as a function of the shell number for our runs G1-8. (Care must be taken to ensure that the coputational runs are sufficiently long to obtain a statistical steady state; see table 1 for our run parameters.) Note that $E(k)$ is plotted on a $\log _{10}$ scale and the shell number is proportional to the logarithm (base 2) of the wavenumber. The slight rise in the curves near $n=1$ is a vestige of the forcing in the first shell. The straight-line portion of 


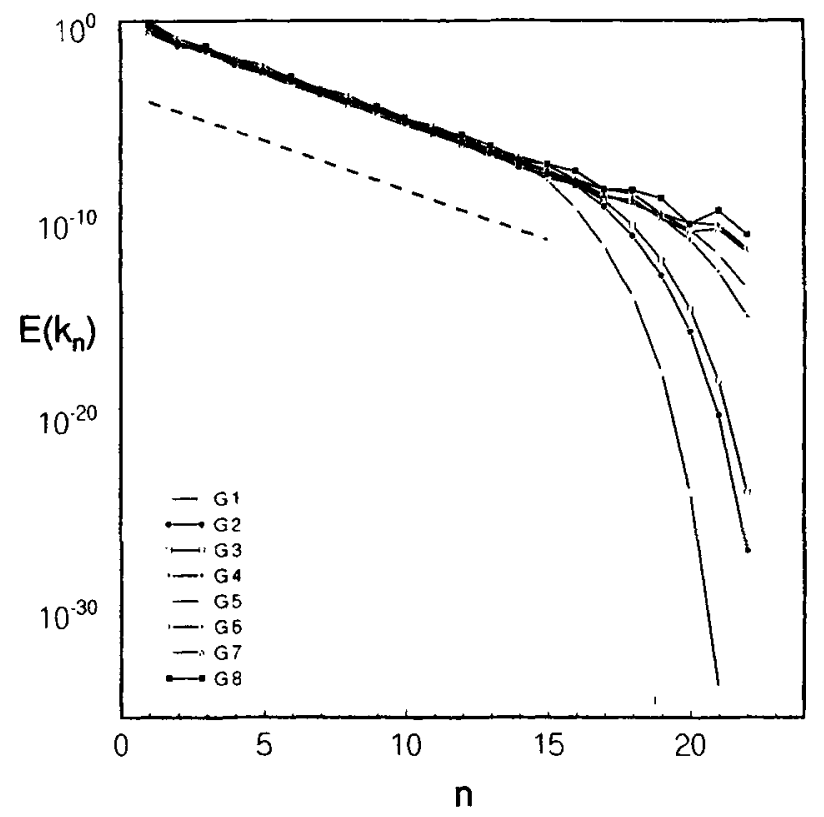

Figure 8. The energy spectrum $E\left(k_{n}\right)$ (plotted on a $\log$ base 10 scale) as a function of shell number $(n)$, for the runs G1-8 (table 1 ). The dashed line represents the $k^{-5 / 3}$ form. The curves for large values of $\nu$ (runs G1-G3) start veering off from the $-5 / 3$ form near the onset of the dissipation range. For small values of $\nu$ (runs G6-8), the dissipation range starts beyond $n=22$.

the plot for intermediate $k$ marks the inertial range and fits the expected $k^{-5 / 3}$ form well. For runs $\mathrm{G} 1-3$, which have the highest viscosities we use, $E(k)$ starts falling from the $k^{-5 / 3}$ line in figure 8. This marks the beginning of the dissipation range. Note that, for the small-viscosity runs (G5-8), only the beginning of this crossover is visible given our wavenumber range. We will return to the form of $E(k)$ in the dissipation range below.

Many authors [82-84] have used such studies to show that GOY-model structure functions exhibit significant deviations from K41 for $p>3$ and, for the conventional choice of parameters which conserve both the energy and the helicity in the inviscid, unforced limit, yield exponents $\zeta_{p}$ in good agreement with experiments and the SL formula. (An approximate analytical understanding of multifractality in the GOY shell model may be obtained by a closure scheme as shown by Benzi, Biferale and Parisi [91].) These studies have also pointed out that $\log$-log plots of $S_{p}\left(k_{n}\right)$ versus $k_{n}$ show an oscillatory behaviour that is a GOY-model artifact related to an underlying 3-cycle in the static solution [92]. In figure 9 we show such a plot for different values of $p$ from our run G1. The increase in $S_{p}$ near $n=1$ arises because we force the first shell. In the region $4 \leq n \leq 14$ the plots are nearly linear, suggesting the algebraic decay of $S_{p}\left(k_{n}\right)$ in the inertial range. The dissipation range starts around $n \simeq 13-17$ for runs G1-G3; this is marked by the bend in the structure functions. The period-three oscillations mentioned earlier show clearly in the inertial range of figure 9 . We have obtained $\zeta_{p}$ 's by fitting the structure functions to a power-law form in the inertial range. As can be seen from figure 15, the fitting errors increase with increasing $p$ because the period-three oscillations are stronger in high-order structure functions. 


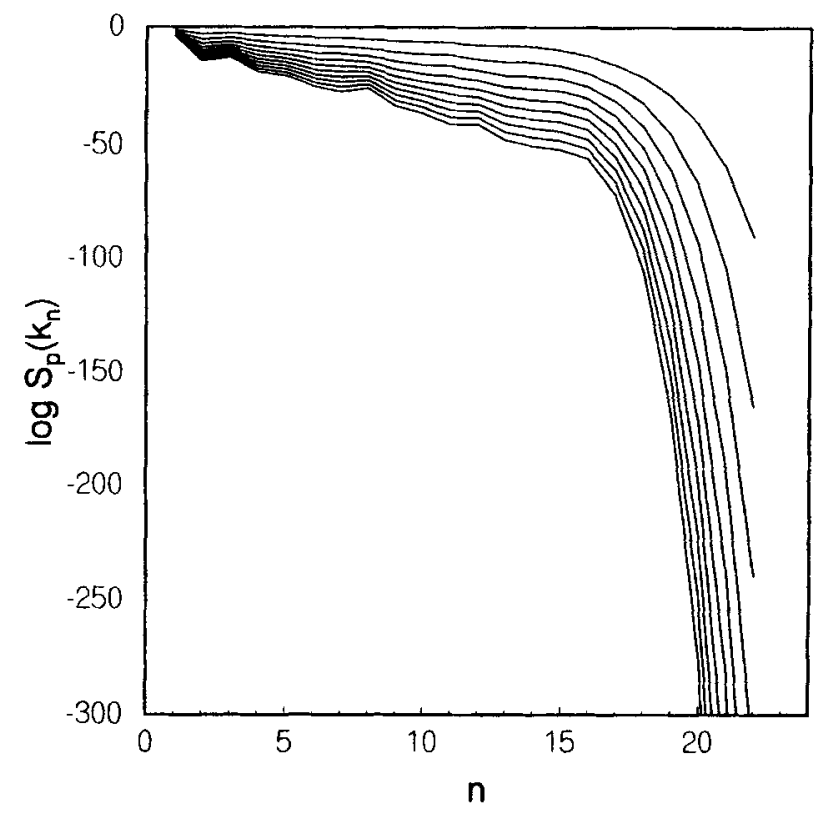

Figure 9. A plot of $\log S_{p}\left(k_{n}\right)$ versus the shell number $n$ for the run $\mathrm{G} 1$ and for $p=2,4,6, \ldots, 20$ (from top to bottom). Note the period-three oscillations that show clearly in high-order structure functions.

Kadanoff et al [84] have pointed out that a simple way of removing these oscillations is to calculate triple moments of velocities; the most effective in removing these oscillations is the structure function

$$
\Sigma_{p}\left(k_{n}\right)=\left\langle\left|\Im\left(v_{n} v_{n+1} v_{n+2}+\frac{1-\delta}{\lambda} v_{n-1} v_{n} v_{n+1}\right)\right|^{p / 3}\right\rangle
$$

which scales like $S_{p}\left(k_{n}\right)$ in the inertial range. Here $\Im$ denotes the imaginary part and we use $\delta=1 / 2$ and $\lambda=2$. In figure 10, we show plots of $\log \Sigma_{p}\left(k_{n}\right)$ against the shell number (n) for our run G1 with $2 \leq p \leq 20$; note that the period-three oscillations of $S_{p}\left(k_{n}\right)$ (figure 9) are absent and the inertial scaling region is a few octaves larger than the one in figure 9. A comparison of the exponents $\zeta_{p}$ calculated from $S_{p}\left(k_{n}\right)$ and $\Sigma_{p}\left(k_{n}\right)$ is shown in figure 11 . The latter not only yields estimates with smaller error bars but also results much closer to the SL prediction (17). Thus we use $\Sigma_{p}\left(k_{n}\right)$ to calculate the $\operatorname{Re}_{\lambda}$ dependence of $\zeta_{p}$ (see below). However, for consistency we use $S_{p}\left(k_{n}\right)$ when we compare our GOY-model results with those of our direct numerical simulation of the $3 d \mathrm{NS}$ equation.

Before presenting our study of $\delta \zeta_{p}$ we note that a similar study has been carried out by using the hierarchical shell model studied by Eggers and Grossmann [95] and Grossmann and Lohse [96]. Their scheme is also referred to as the reduced wavevector approximation (REWA). The model considers the velocity field associated with the $n$-th shell to be given by a set of variables $v_{n, j}$, where $j=1, \ldots, J^{n}$, with $J>1$. Each variable $v_{n, j}$ interacts with all the variables in the $n \pm 1$ and $n \pm 2$ shells. The resulting set of 


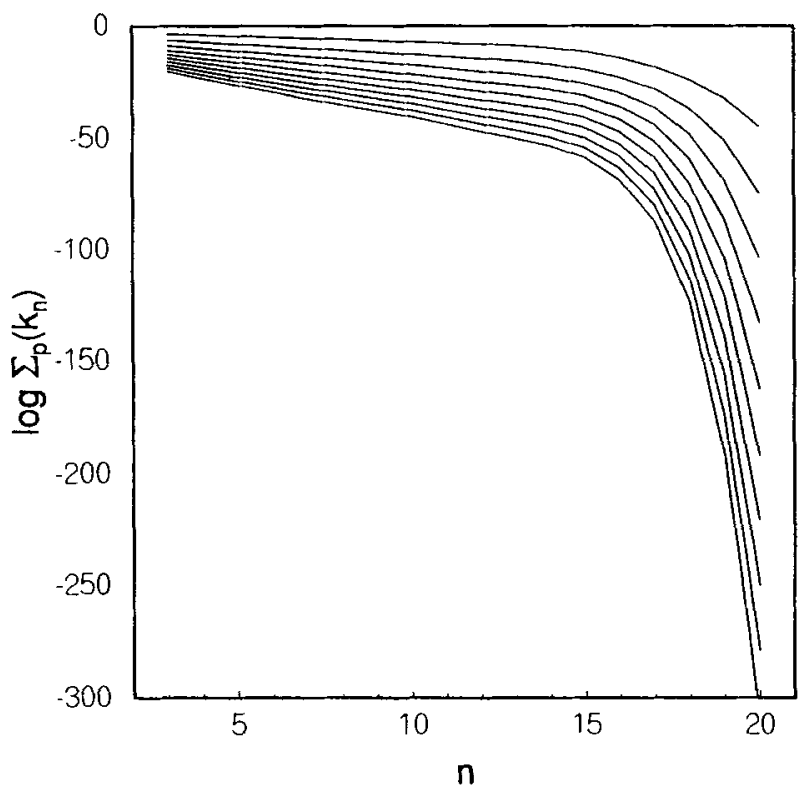

Figure 10. A plot of $\log \Sigma_{p}\left(k_{n}\right)$ (see text) versus the shell number $n$ for the run G1 and for $p=2,4,6, \ldots, 20$ (from top to bottom). Note that the curves are free from the oscillations that appear in the structure-function plots (figure 9).

differential equations has many more nonlinear terms than its analogue in the GOY model. The advantage of this added complexity is that the nonlinear term has a greater similarity with its counterpart in the NS equation (26). The REWA model has many more degrees of freedom than the GOY model, so its numerical solution is almost as difficult as a direct solution of the $3 d$ NS equation. Of course, much higher values of Re can be achieved for the REWA models than for the $3 d$ NS equation. We refer the reader to refs $[8,95,96]$ for details of this model. Grossmann, Lohse, L'vov and Procaccia [35] have used the REWA model to cover the range $10^{4}<\operatorname{Re}<10^{7}$. (In these simulations, $\operatorname{Re}_{\lambda} \sim 12 \sqrt{\operatorname{Re}}$ as shown in ref. [96].) From their plots of $\delta \zeta_{p}=\left|\zeta_{p}-p / 3\right|$ versus $\operatorname{Re}$, these authors argue that the theoretical prediction of ref. [36], namely, $\delta \zeta_{p} \sim \operatorname{Re}^{-3 / 10}$, might well be true as $\operatorname{Re} \rightarrow \infty$.

We have tried to address this issue for the GOY shell model. Specifically, we have obtained $\zeta_{p}$ and hence $\delta \zeta_{p}$ from $\Sigma_{p}\left(k_{n}\right)$ for eight different Reynolds numbers (runs G1-8, table 1) in the range $4 \times 10^{4} \lesssim \operatorname{Re}_{\lambda} 3 \times 10^{6}$. The resulting data are shown in the plot of $\delta \zeta_{p}$ versus $\operatorname{Re}_{\lambda}$ in figure 12 ; the SL prediction is also shown for some values of $p$. There seems to be no indication of a decrease in $\delta \zeta_{p}$ with increasing $\operatorname{Re}_{\lambda}$; if anything, there is a marginal increase. (We have checked that the increase in $\delta \zeta_{p}$ at the highest value of $\operatorname{Re}_{\lambda}$ (run G8) remains on choosing a smaller time step and larger averaging time for this run.) This result is in contrast with the conclusion of Grossmann et al [96]. It is possible that this discrepancy arises because of some feature that is present in the REWA model but not in the GOY shell model. Only more exhaustive numerical simulations (ideally of the $3 d$ NS equation) and systematic experimental studies will be able to decide whether the trend shown in figure 12 actually obtains in fluid turbulence. We also note that, on the basis of their static analysis of the GOY model, Schörghofer, Kadanoff, and Lohse [92] have

Pramana - J. Phys., Vol. 48, No. 1, January 1997 (Part I) 

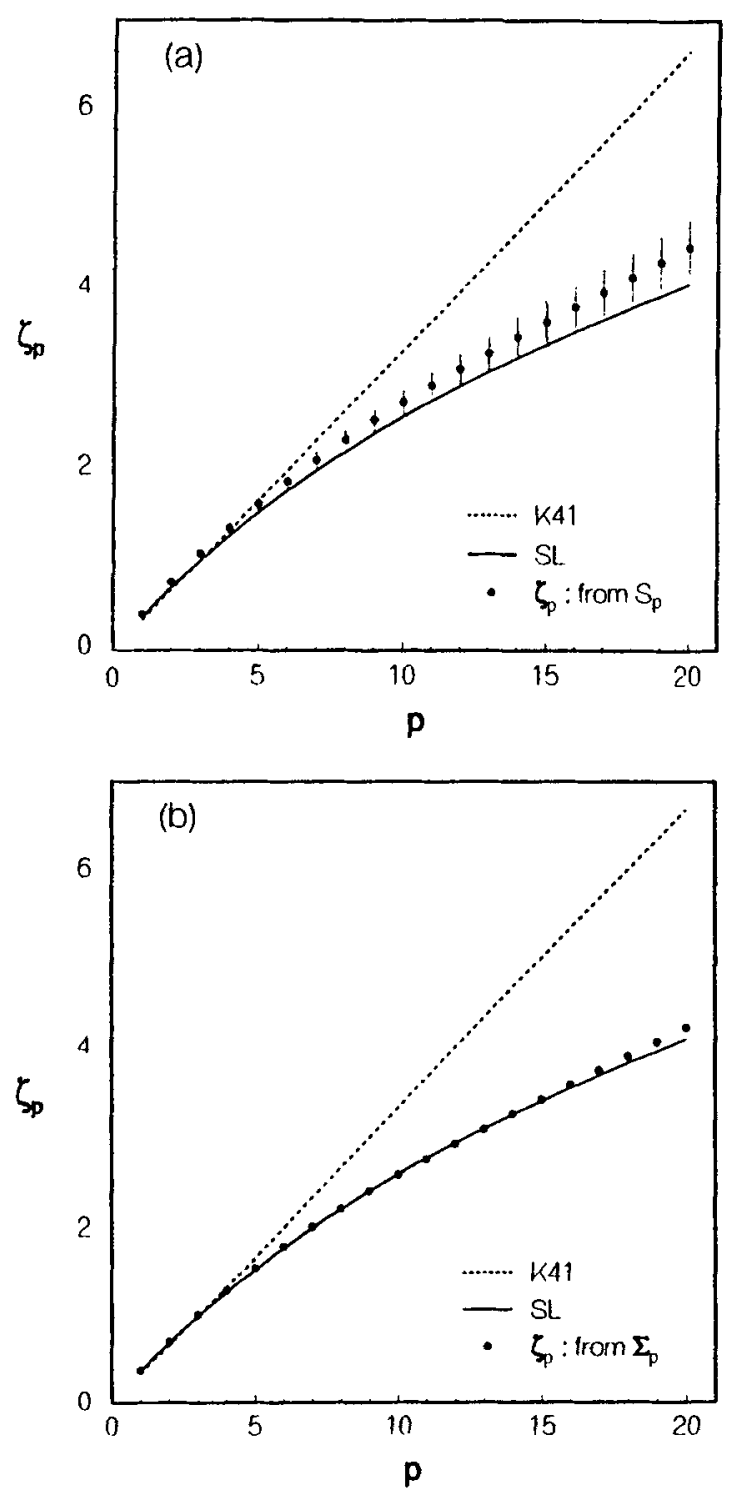

Figure 11. $\zeta_{p}$ versus $p$ for our run $\mathrm{Gl}$ obtained from the best straight-line fit in the inertial range by using (a) $S_{p}\left(k_{n}\right)$ and (b) $\Sigma_{p}\left(k_{n}\right)$. Error bars in (a) denote the fitting errors; similar error bars are also shown in (b) but are not apparent since they are comparable to the symbol sizes. The K41 and the SL predictions are also shown.

suggested that $\zeta_{p}$ should have an oscillatory dependence on $\log \nu$; our fully dynamical calculation (figure 12) seems to be in agreement with this suggestion.

The obvious question that arises is whether the $\zeta_{p}$ 's are universal. The data of figure 12 suggest they are not. This has also been found by Leveque and She [97] in their study of the GOY model in the presence of pure hyperviscosity, i.e., with a $-\nu_{H} k^{\alpha_{H}}$ dissipation and $\alpha_{H}$ not necessarily 2 : their $\zeta_{p}$ 's depend on $\alpha_{H}$. Similar results have been obtained by 


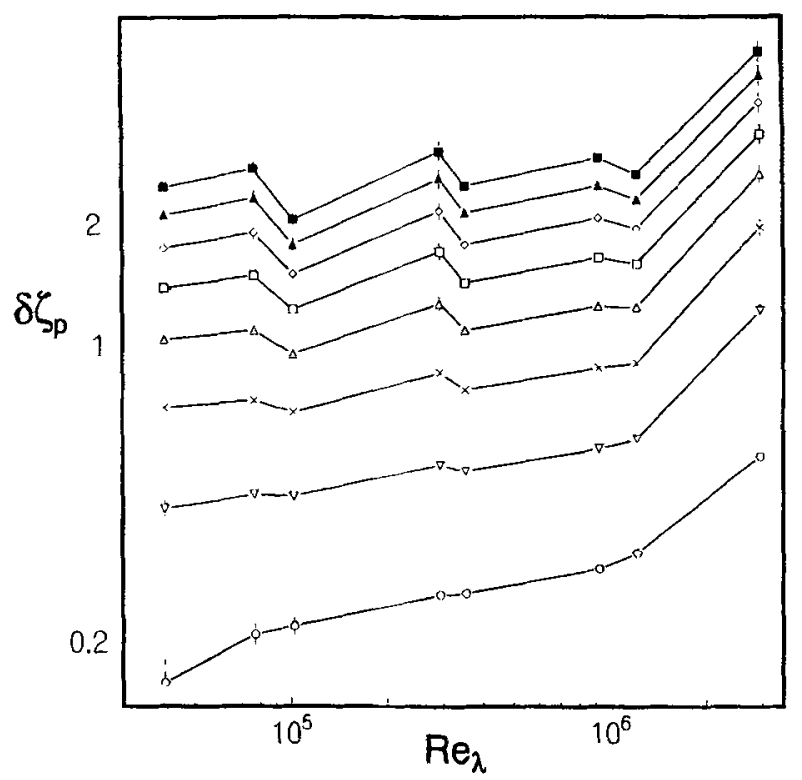

Figure 12. Log-log plot of $\delta \zeta_{p}$ versus the Taylor-microscale Reynolds number $\operatorname{Re}_{\lambda}$ for our GOY-model runs (G1-8) with $p=6,8, \ldots, 20$ (from bottom to top). The dotted $(p=6)$ and dashed $(p=8)$ lines show the SL results. Error bars are shown but are often smaller than the symbol sizes.

Borue and Orszag [98] in a direct numerical solution of the NS equation with pure hyperviscosity. However, this nonuniversality is removed [97] if one uses the ratios $\zeta_{p} / \zeta_{3}$. We shall return to this point in our discussion of $k$-space ESS and GESS below.

Before presenting our numerical studies of ESS and GESS, we wish to discuss the form of $E(k)$ in the dissipation range. It has been suggested by some authors $[60,99]$ that the energy spectrum in the far-dissipation range is of the form

$$
E(k) \sim f\left(k / k_{\mathbf{d}}\right) \exp \left[-c\left(k / k_{\mathbf{d}}\right)^{n}\right],
$$

where $c$ is a constant, $f$ is a weak function of $k / k_{\mathrm{d}}$, and $1 \leq n \leq 2$. In their direct numerical simulation of the NS equation, Chen et al [59] find that the form

$$
E(k) \sim k^{\alpha} \exp \left(-c k / k_{\mathrm{d}}\right)
$$

is consistent with their data with $\alpha \simeq 3.3$ and $c \simeq 7.1$. This pseudo-spectral simulation uses $256^{3}$ Fourier modes and has achieved the best numerical resolution of the far dissipation range of the $3 d$ NS equation to date. Our low- $\operatorname{Re}_{\lambda}$ studies (table 1 ) also yield a dissipation range in which $E(k)$ fits such a form. We have also checked explicitly that our data for $S_{3}$ in the GOY model can be fit to the form

$$
S_{3} \sim k^{\delta} \exp \left(-c k / k_{\mathrm{d}}\right)
$$

in the dissipation range (run G1) with $\delta=3.80$ and $c / k_{\mathrm{d}}=4.13 \times 10^{-4}$. (We note that such fits are difficult in the GOY model and may not be unique: given the logarithmic sampling of $k$ space, we have only five points in five octaves of $k$.) 

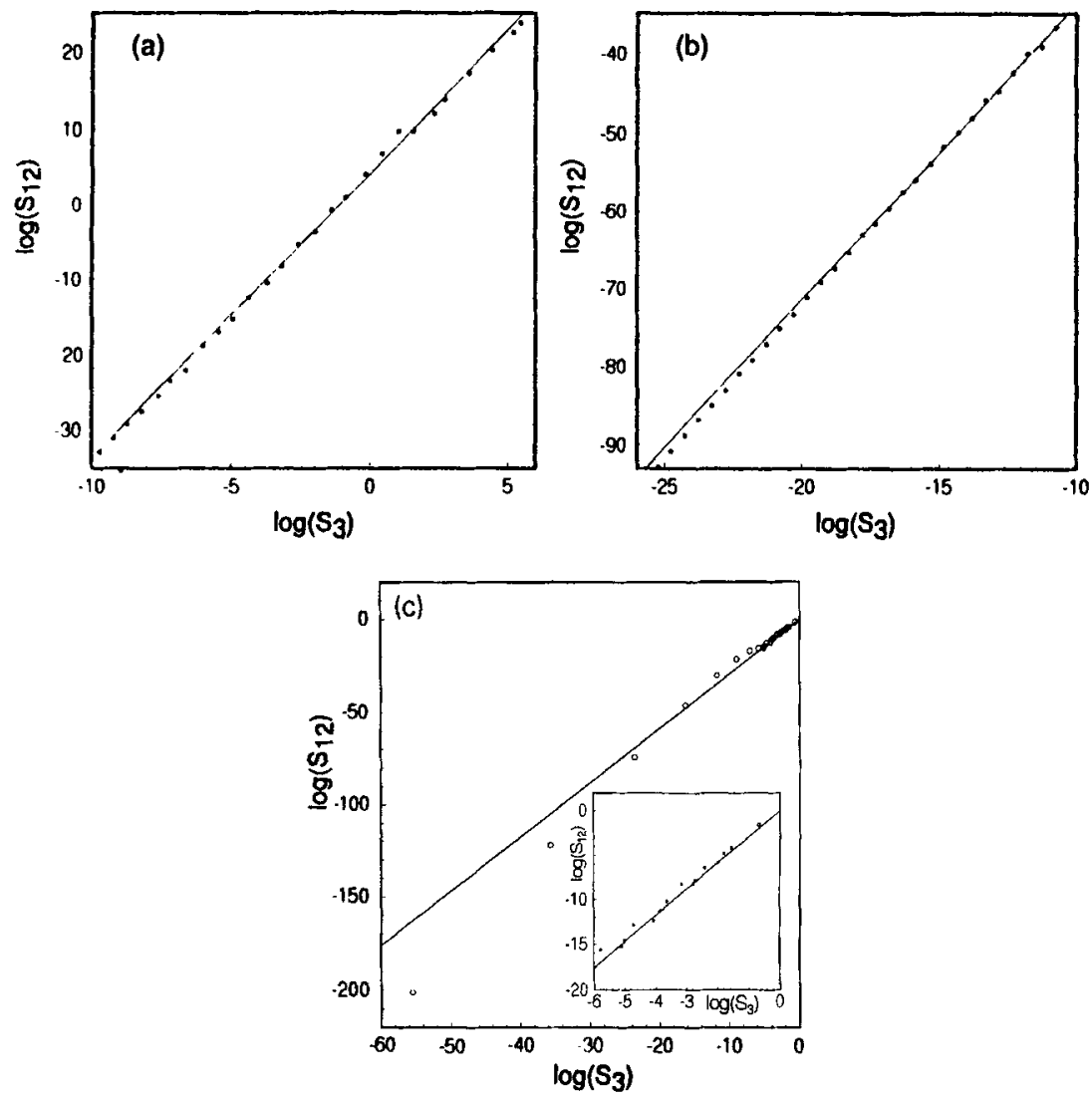

Figure 13. Log-log plots for $S_{12}(k)$ versus $S_{3}(k)$ for our runs (a) NS1 (the inertial range), (b) NS1 (the dissipation range) and (c) G1. The inset in (c) shows a magnified view of data in the inertial range. The straight lines represent $S_{12} \sim S_{3}^{\zeta_{p}^{S L}}$. Deviations from this line show clearly for data in the dissipation range (large $k$, i.e., small $S_{3}$ ).

In recent work three of us [89] have proposed and verified a $k$-space version of ESS and GESS in the inertial range. We have also extended the applicability of ESS and GESS to the far dissipation range to uncover intriguing and apparently universal crossover from inertial to dissipation ranges. Our $k$-space ESS postulate is

$$
\begin{gathered}
S_{p} \equiv\left\langle|\mathbf{v}(\mathbf{k})|^{p}\right\rangle \approx A_{I p}\left(S_{3}\right)^{\zeta_{p}^{\prime}}, \quad L^{-1} \ll k \lesssim 1.5 k_{\mathrm{d}}, \\
S_{p} \equiv\left\langle|\mathbf{v}(\mathbf{k})|^{p}\right\rangle \approx A_{D p}\left(S_{3}\right)^{\alpha_{p}}, \quad 1.5 k_{\mathrm{d}} \lesssim k \ll \Lambda,
\end{gathered}
$$

where the $A_{I p}$ and $A_{D p}$ are, respectively, amplitudes for inertial and dissipation ranges and $\Lambda^{-1}$ the (molecular) length at which hydrodynamics fails. Note that our postulate suggests different power-law behaviours in inertial and dissipation ranges; the real-space analogue of this was noted by Stolovitzky and Sreenivasan [22], who used experimental data, soon after the proposal of real-space ESS by Benzi, et al [32]. We do find that this way of presenting the data extends the apparent inertial range in both our NS and GOYmodel studies, so the expression $k$-space ESS is justified. Representative log-log ESS 


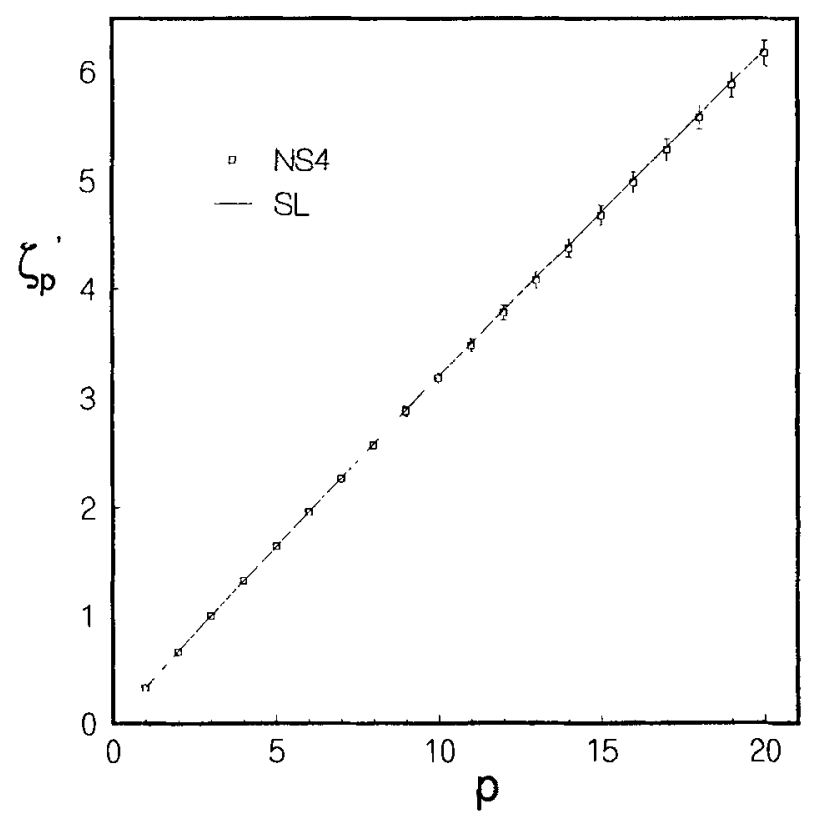

Figure 14. A plot of $\zeta_{p}^{\prime}$ versus $p$. The squares indicate data from our run NS4. The line is $\zeta_{p}^{\prime}=\left(2 \zeta_{p}+3 p\right) / 11$, with $\zeta_{p}=\zeta_{p}^{\mathrm{SL}}$ (see text).

plots of $S_{12}(k)$ versus $S_{3}(k)$ from our run NS1 (table 1) are shown in figure 13: here panel (a) covers the inertial range and yields the inertial-range power $\zeta_{12}^{\prime}$, which is in agreement with the SL prediction; panel (b) covers the dissipation-range and yields a power $\alpha_{12}$, which deviates noticeably from the SL prediction; panel (c) illustrates the same crossover from inertial- to dissipation-range asymptotic behaviours for the GOY shell model, where we can resolve much larger inertial and dissipation ranges, so this crossover shows clearly. Note also that the period-3 oscillations in figure 9 (structure functions for the GOY model) are removed in such ESS plots, yielding better estimates for the exponents. The agreement between our NS and GOY results, both qualitative and quantitative (see below), indicates that this crossover is real and not a numerical artifact. To ensure that we have good statistics, all our NS runs have been done in quadruple precision and we have checked that our estimates for $\zeta_{p}^{\prime}$ and $\alpha_{p}$ do not change noticeably when we double our averaging time or halve our integration time step. Our NS runs are such that NS1, NS2, and NS3 resolve enough of the dissipation range (table 1) to yield dissipation-range exponents $\alpha_{p}$; run NS4 does not yield many points in the dissipation range, so we do not use it to obtain $\alpha_{p}$.

In (41) the inertial-range exponent: $\zeta_{p}^{\prime}=\zeta_{p}$, for the GOY model; however, we find explicitly (figure 14) that, for the $3 d$ NS case,

$$
\zeta_{p}^{\prime}=\left(\zeta_{p}+3 p / 2\right) /\left(\zeta_{3}+9 / 2\right)
$$

whence we get (36), i.e., $S_{p}(k) \sim k^{-\left(\zeta_{p}+3 p / 2\right)}$, in the inertial range. To our knowledge this result for $S_{p}(k)$ is new. Our NS runs, though restricted to relatively low $\operatorname{Re}_{\lambda}(\lesssim 22)$, obtain it via ESS (41) and $\zeta_{3}=1$. For even values of $p$ we can obtain this result via

Pramana - J. Phys., Vol. 48, No. 1, January 1997 (Part I) 


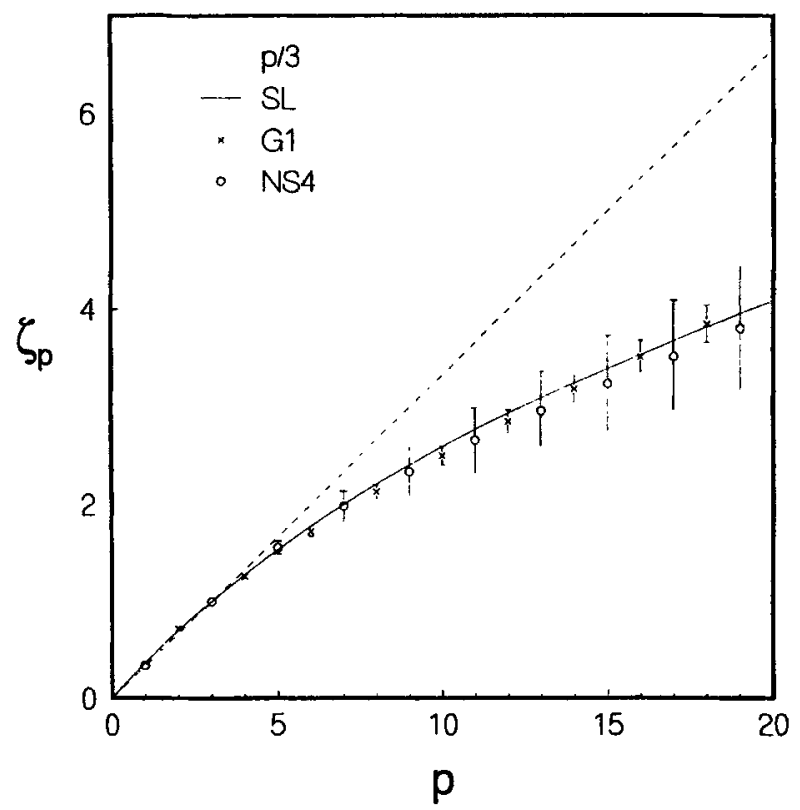

Figure 15. A plot of $\zeta_{p}$ versus $p$ for the runs NS4 and G1. The K41 and SL lines are also shown. For NS4 we obtain $\zeta_{p}$ by inverting (47) and using our data for $\zeta_{p}^{\prime}$; this inversion also magnifies the error bars (cf. figure 14).The estimates for these exponents are obtained from ESS plots like figure 13 for both GOY and NS runs.

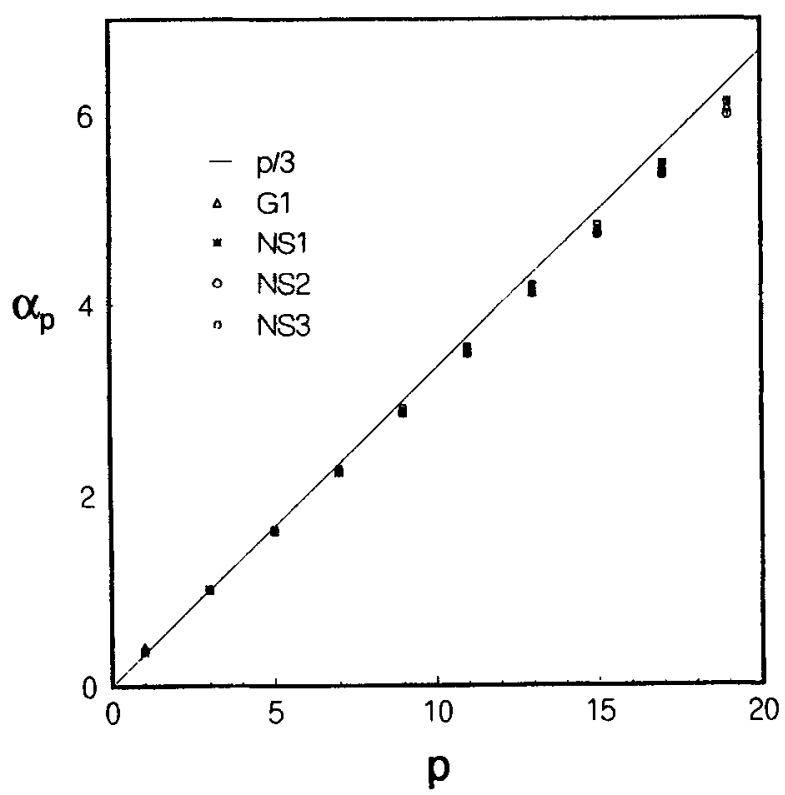

Figure 16. A plot of $\alpha_{p}$ versus $p$ for the runs NS1-3 and G1. The K41 line is also shown. The estimates for $\alpha_{p}$ are obtained from the dissipation-range asymptotes of ESS plots like figure 13.

Pramana - J. Phys., Vol. 48, No. 1, January 1997 (Part I) 


\section{Sujan K Dhar et al}

dimensional analysis if we Fourier transform the real-space structure function $\mathcal{S}_{p}$ and make one numerically plausible assumption: namely, that $\left\langle\mathbf{v}_{i 1}\left(\mathbf{k}_{1}\right), \ldots, \mathbf{v}_{i p}\left(\mathbf{k}_{\mathrm{p}}\right)\right\rangle$ is dominated by terms in which the $\mathbf{k}_{m}, m=1, \ldots, p$, arguments form equal and opposite pairs all with magnitude $k$, i.e., $\left\langle\mathbf{v}_{i 1}\left(\mathbf{k}_{1}\right), \ldots, \mathbf{v}_{i p}\left(\mathbf{k}_{\mathbf{p}}\right)\right\rangle \sim S_{p}(k)\left[\delta\left(\mathbf{k}_{1}+\mathbf{k}_{2}\right) \ldots\right.$ $\delta\left(\mathbf{k}_{p-1}+\mathbf{k}_{p}\right)+$ permutations $]$.

Both $\zeta_{p}$ (figure 15) and $\alpha_{p}$ (figure 16) seem universal, to the extent that, given our error bars, they are the same for all our GOY-model and $3 d$ NS runs (table 1). $\zeta_{p}$ agrees reasonbly well with the She-Leveque (SL) formula [51] for the ranges of $p$ and $\operatorname{Re}_{\lambda}$ in figure 15; and $\alpha_{p}$ is close to, but systematically less than, $p / 3$. Of course, figure 12 indicates that the GOY-model exponents have a mild depedence on $\nu$; however, as noted above, this nonuniversality is removed in our ESS and GESS plots (see below) in which only ratios of the exponents $\zeta_{p}$ appear.

Given our $k$-space ESS (41), the asymptotic $k$ dependences of $S_{p}(k)$ in inertial and dissipation ranges follow from the dependence of $S_{3}$ on $k$. We find

$$
\begin{aligned}
& S_{3} \approx B_{I} k^{-\zeta_{3}-9 / 2}, \quad L^{-1} \ll k \lesssim 1.5 k_{\mathrm{d}}, \\
& S_{3} \approx B_{D} k^{\delta} \exp \left(-c k / k_{\mathrm{d}}\right), \quad 1.5 k_{\mathrm{d}} \lesssim k \ll \Lambda,
\end{aligned}
$$

where $B_{I}$ and $B_{D}$ are, respectively, nonuniversal amplitudes. (Equation (44) holds for the $3 d$ NS equation (see above); for the GOY model the factor $9 / 2$ is absent.) This implies that, in the far dissipation range,

$$
S_{p} \sim k^{\theta_{p}} \exp \left(-c \alpha_{p} k / k_{\mathrm{d}}\right), \quad k_{\mathrm{d}} \ll k \ll \Lambda,
$$

with $\theta_{p}=\alpha_{p} \delta$, for all $p$. This form is not easy to verify numerically for large $p$, given the rapid decay at large $k$. To the best of our knowledge it has been suggested so far [59] only for $S_{2}$.

In (44), $\delta, c$, and $k_{\mathrm{d}}$ are not universal. Our $k$-space ESS shows that there is a universal part to the inertial- to dissipation-range crossover. We now show that this universal crossover can be extracted best by a $k$-space version of GESS that three of us have proposed recently [89]. This holds for both the GOY model and the NS equation. (Realspace GESS, due to Benzi et al [32], has been discussed in $\S 2$.) We begin by defining

$$
G_{p} \equiv S_{p} /\left(S_{3}\right)^{p / 3}
$$

Log-log plots of $G_{p}$ versus $G_{q}$ yield curves with asymptotes which have universal, but different, slopes in inertial and dissipation ranges. Figure 17 shows a representative plot, with both GOY-model and NS data, for $p=6$ and $q=9$. The inertial-range asymptote has a slope $\rho(p, q)$ given by $(21)$; it is easy to check from the formulae above that this is the same as the slope of real-space GESS plots [58] in the inertial range. From the SL value [51] for $\zeta_{p}(17)$ we can obtain the SL prediction for $\rho(p, q)$; the resulting inertialrange asymptote is in good agreement with our data in the inertial-range part of figure 17. From our $k$-space ESS and GESS ansätze above, it also follows that the dissipation-range asymptote has a slope

$$
\omega(p, q) \equiv\left[\alpha_{p}-p / 3\right] /\left[\alpha_{q}-q / 3\right],
$$

where $\alpha_{p}$ is defined in (41) and shown in figure 16. Note that GESS plots amplify

Pramana - J. Phys., Vol. 48, No. 1, January 1997 (Part I) 

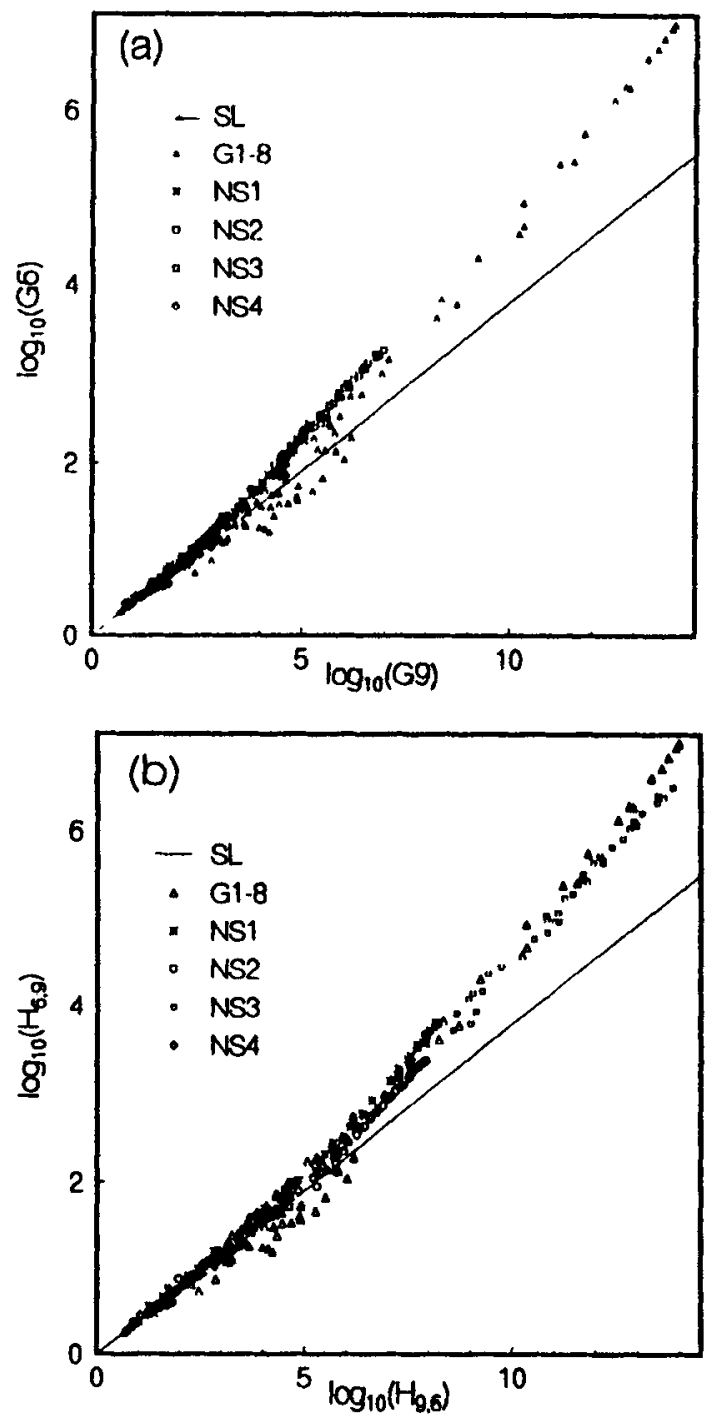

Figure 17. Log-log plots for (a) $G_{6}$ versus $G_{9}$, and (b) $H_{9,6}$ versus $H_{6,9}$ (see text) for all our NS and GOY-model runs. The data collapse in (b) yields the universal scaling function that characterizes the crossover from the inertial to the dissipation range, for $p=6$ and $q=9$ here.

considerably the difference between inertial- and dissipation-range asymptotes, which is not very prominent in ESS plots; this amplification can be estimated analytically.

In GESS plots like figure 17 the slopes of these inertial- and dissipation-range asymptotes are universal, at least at the level of accuracy of figures 15 and 16; but the point at which the curve in a GESS plot starts deviating from the inertial-range asymptote depends on the model (GOY, NS, with and without hyperviscosity, etc.). It is easily seen, though, that the following transformation yields a universal crossover scaling function (this curve is, of course, different for each pair of values of $p$ and $q$ because of

Pramana - J. Phys., Vol. 48, No. 1, January 1997 (Part I) 


\section{Sujan $K$ Dhar et al}

multiscaling): We define

$$
\begin{aligned}
& \log \left(H_{p q}\right) \equiv D_{p q} \log \left(G_{p}\right), \\
& \log \left(H_{q p}\right) \equiv D_{q p} \log \left(G_{q}\right),
\end{aligned}
$$

where the scale factors $D_{p q}=D_{q p}$ are nonuniversal. Now plots of $\log \left(H_{p q}\right)$ versus $\log \left(H_{q p}\right)$ show data collapsing onto one universal curve within our error bars. This is illustrated in figure $17 \mathrm{~b}$ for $p=6$ and $q=9$; we emphasize that the data collapse occurs for all GOY and $3 d$ NS runs and for all $k$ and $\mathrm{Re}_{\lambda}$. In essence this transformation holds one of the GESS plots fixed and slides those of other models till the asymptotes match.

In their discussion of real-space GESS, Benzi et al have suggested a hierarchy for the $\mathcal{G}_{p}(r)$, which leads to the SL formula if one uses the SL boundary conditions mentioned above. This hierarchy has a $k$-space analogue [89], namely

$$
\left[G_{p+1} / G_{p}\right]=\left[G_{p} / G_{p-1}\right]^{\gamma} \times\left[\lim _{p \rightarrow \infty} G_{p+1} / G_{p}\right]^{1-\gamma}
$$

with $\gamma^{3}=2 / 3$. If we make the additional assumption $G_{p}(k) \sim k^{\beta_{p}}$, which holds in the inertial range, we get a difference equation for $\beta_{p}$ identical to the SL one, which, when solved with the boundary conditions $\beta_{0}=\beta_{3}=0$ and $\lim _{p \rightarrow \infty}\left(\beta_{p+1}-\beta_{p}\right)=2 / 9$, yields the SL formula (17) via (7) (our $\beta_{p}=-\tau_{p / 3}$ there). Elementary manipulations show that our $k$-space GESS gives $\left[G_{p+1} / G_{p}\right] \sim\left[G_{p} / G_{p-1}\right]^{\Upsilon_{p}}$, with $\Upsilon_{p}=\left(\zeta_{p+1}-\zeta_{p}-1 / 3\right) /$ $\left(\zeta_{p}-\zeta_{p-1}-1 / 3\right)$. At first sight this seems inconsistent with the assumed hierarchy (49); however, it can be seen to be consistent if $\Upsilon_{p}=\gamma-2(1-\gamma) /\left[9\left(\zeta_{p}-\zeta_{p-1}-\zeta_{3} / 3\right)\right]$, which turns out to be the SL difference equation. Thus we cannot claim to have checked either of the assumed hierarchies (14) or (49) directly. Our $k$-space GESS can certainly hold with $\zeta_{p} \neq \zeta_{p}^{\mathrm{SL}}$. We can only claim that the hierarchy might hold to the extent that our calculated values of $\zeta_{p}$ agree reasonably with those predicted by the SL formula (figure 15).

The difference between inertial- and dissipation-range asymptotic behaviours has not been noted in real-space GESS so far. As we have mentioned above, experimental evidence for slope differences between inertial- and dissipation-range asymptotes in real-space ESS plots was given by Stolovitzky and Sreenivasan [22]. They postulated $\mathcal{S}_{p} \sim \mathcal{S}_{3}^{\alpha_{p}^{\prime}}$ in the dissipation range and suggested

$$
\alpha_{p}^{\prime} \simeq \frac{\zeta_{3 p / 2}+p / 2}{\zeta_{9 / 2}+3 / 2} .
$$

Unfortunately, we have not been able to find a simple, direct relation between their realspace exponents $\alpha_{p}^{\prime}$ and our $k$-space exponents $\alpha_{p}$ (unlike (42) for inertial-range exponents), since $S_{p}$ does not have a simple power-law dependence on $k$ in the dissipation range. Furthermore, in this range, $G_{p} \sim k^{\beta_{p}^{\prime}} \exp \left(-c_{p}^{\prime} k / k_{\mathrm{d}}\right)$, so there is no analogue of the SL formula for our dissipation-range exponents $\alpha_{p}$. Nonetheless it should be interesting to study the apparently universal inertial- to dissipation-range crossover that we have elucidated above in experimental flows. 


\section{Homogeneous isotropic turbulence}

\subsection{Probability distributions}

Direct numerical simulations have obtained data for various probability ditributions $[64,73]$. In these studies, which have attained $\operatorname{Re}_{\lambda}=150$, it has been found that the distribution of velocity components is close to Gaussian. However, the distributions of velocity derivatives such as $\partial_{x} \mathbf{v}_{x}$ show significant deviations from Gaussian behaviour, which are consistent with experimental results. This deviation is even more visible in the distribution of derivatives like $\partial_{y} \mathbf{v}_{x}$. The PDFs of such velocity derivatives show tails that decay even more slowly than tails of exponential distributions.

PDFs of velocity differences have also been obtained by these direct numerical studies [73]. The distributions found are similar to those seen in experiments $[31,62]$ and seem to have exponential tails for small separtions $r$; as this separtion increases, the deviations from Gaussian character decrease till, at large separtions comparable to integral scales, these PDFs approach Gaussians.

These simulations [73] have tried to check the log-normal hypothesis of Kolmogorov [42] for the distribution of the energy dissipation rate $\epsilon$. Though there is reasonable agreement near the peak of the distribution, there are significant deviations from this form in the tails. We are not aware of any direct numerical checks of the log-Poisson class of distributions that follow from the She-Leveque assumptions [55,67]. Data from our Navier-Stokes runs would not be good enough to rule out other possible distributions. To obtain information about the distribution of $\epsilon$ in the GOY model, one must transform to real space. As we have said earlier, the logarithmic discretisation of $k$ space makes such transformation questionable for this model, so we restrict ourselves to the distributions of Fourier components (see below). The best we can do is to check the analogue of She-Leveque hierarchy for the moments of $\epsilon$, which implies a distribution of the log-Poisson class. We have discussed this above in terms of $G_{p}$ (see the paragraph with (49)).

The study of probability distributions in shell models is of more recent origin. Non-Gaussian behaviour in the GOY shell model has been studied by Biferale [100]. He has shown, by taking a Fourier transform of $v_{n}$, that PDFs of the realspace velocity gradients are non-Gaussian for the GOY shell model and not far from the predictions of a multifractal theory. Also, the deviations from Gaussian behaviour increase with decreasing spatial separation, in qualitative accord with the results for the NS equation and from experiments. One might question whether Fourier transforms over the logarithmically spaced wavenumbers in the GOY model can give reasonable real-space information. Thus, in our study, we restrict ourselves to $k$ space quantities and look for possible non-Gaussian statistics. The REWA models sample $k$-space better than the GOY model. These have also been used to obtain the PDFs of velocity differences [101] which show a crossover from Gaussian behaviour, at the largest spatial scales, to PDFs with increasingly stretched tails at smaller scales.

In analogy with the experimental studies of Katsuyama $e t$ al we first construct $k$-space $\gamma_{2 p}$ 's (cf. (23)) for the GOY shell model:

$$
\gamma_{2 p}=\frac{(2 p-1) ! !}{2} \frac{\left\langle\left|v_{n}\right|^{2}\right\rangle^{p}}{\left\langle\left|v_{n}\right|^{2 p}\right\rangle}, \quad p=2,3,4, \ldots,
$$



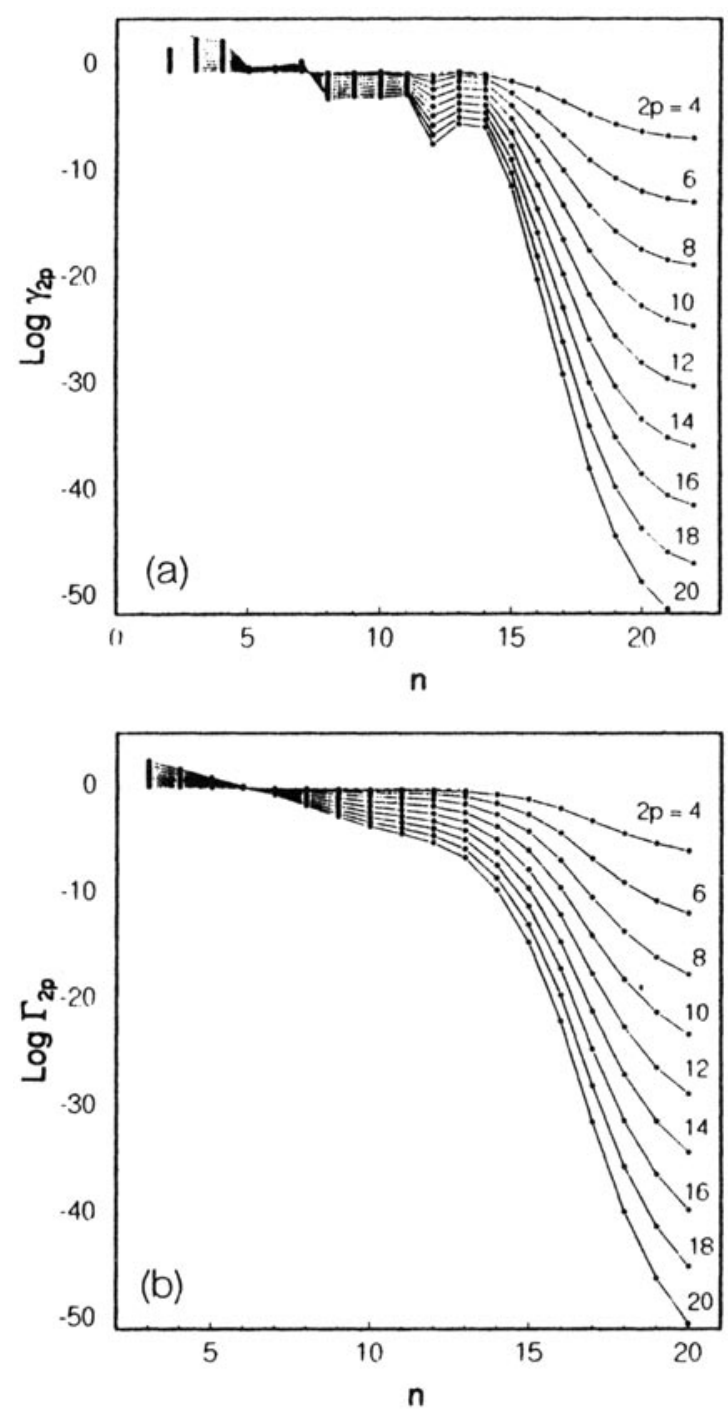

Figure 18. Plots of (a) $\gamma_{2 p}$ and (b) $\Gamma_{2 p}$ versus the shell number $n$ for $2 p=4,6, \ldots, 20$, for our run G1. The dashed lines indicate $\gamma_{2 p}=0.5$ and $\Gamma_{2 p}=0.5$, respectively. One can see that the plots in (b) are free from the oscillations present in (a).

which can be rewritten as

$$
\gamma_{2 p}=\frac{(2 p-1) ! !}{2} \frac{S_{2}^{p}}{S_{2 p}} \quad p=2,3,4, \ldots
$$

Note that the midband frequency $f_{c}$ in (23) has been replaced, effectively, by the shell number $n$ here. In figure $18 \mathrm{a}$ we plot $\gamma_{2 p}$ (calculated via eq. (52)) as a function of $n$, the shell number, for run G1. One can clearly observe the deviation of $\gamma_{2 p}$ from the Gaussian 

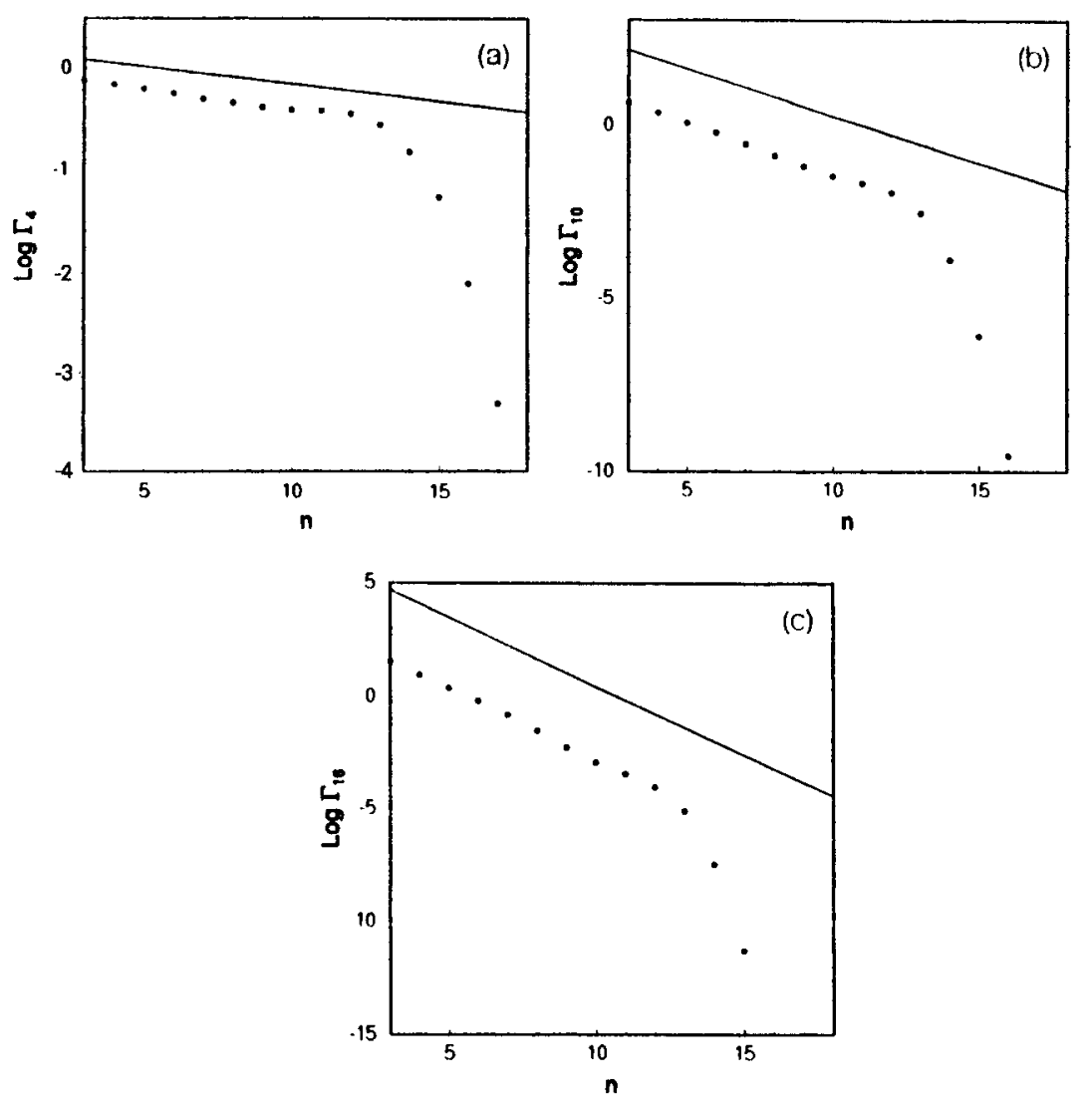

Figure 19. Inertial-range behaviour of (a) $\Gamma_{4}$ (b) $\Gamma_{10}$ and (c) $\Gamma_{16}$ for run G1 (figure 18). Continuous lines represent the form $\Gamma_{2 p} \propto \frac{1}{2}(2 p-1) ! ! k_{n}^{\zeta_{2 p}-p \zeta_{2}}$, with the $\zeta_{p}$ 's calculated from the SL formula.

value of 0.5 in the inertial range and the rapid decay of $\gamma_{2 p}$ in the dissipation range (beyond $n=14$ ). However, the curves show oscillations that arises because of the underlying 3-cycle in the GOY shell model (see above). To eliminate these we define a new function $\Gamma_{2 p}$ in terms of $\Sigma_{p}((37))$ :

$$
\Gamma_{2 p}=\frac{(2 p-1) ! !}{2} \frac{\Sigma_{2}^{p}}{\Sigma_{2 p}}, \quad p=2,3,4, \ldots
$$

Figure $18 \mathrm{~b}$ shows the variation of $\Gamma_{2 p}$ (calculated using (53)) with $n$ for run G1. As one might have anticipated, the use of the correlation function $\Sigma_{p}$ has removed the oscillations so the deviations from Gaussian behaviour show clearly.

Since we know that $\Sigma_{p} \sim k_{n}^{-\zeta_{p}}$ in the inertial range, we can infer from (53) the form of $\Gamma_{2 p}$ in the inertial range, i.e.,

$$
\Gamma_{2 p} \propto \frac{(2 p-1) ! !}{2} k_{n}^{\zeta_{2 p}-p \zeta_{2}}, \quad L^{-1} \ll k \lesssim 1.5 k_{\mathrm{d}}
$$

To specify $\Gamma_{2 p}$ completely in the inertial range we must also provide the amplitudes for the power-law dependence of $\Sigma_{p}$ on $k$. However, this is not necessary for the purpose 

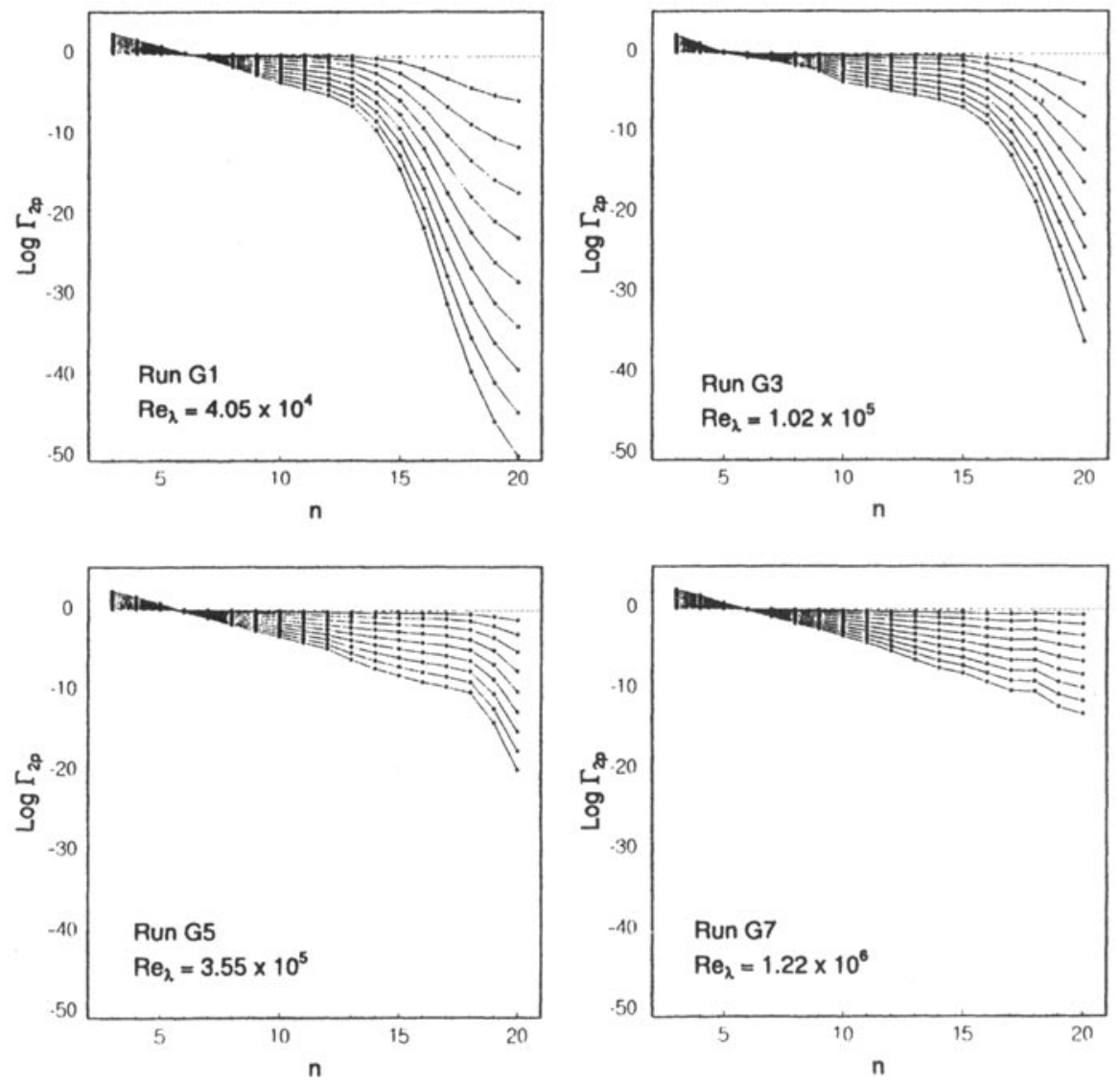

Figure 20. $\Gamma_{2 p}$ as functions of the shell number $n$ for our runs G1, G3, G5, and G7 for $2 p=4,6, \ldots, 20$ (top to bottom). Dashed lines indicate $\Gamma_{2 p}=0.5$.

of figure 19, where we show comparisons between the $\Gamma_{2 p}$ 's shown in figure $18 \mathrm{~b}$ and the form suggested in (54) (with the $\zeta_{p}$ 's calculated from the SL formula) for $2 p=4,10$ and 16. Clearly, similar power laws obtain in the inertial range. We note in passing that the slight hump before the dissipation range is an indication of the bottleneck phenomenon [61].

Figure 20 shows $\Gamma_{2 p}$ as a function of the shell number $n$ for four different $\operatorname{Re}_{\lambda}$ (our runs G1, G3, G5 and G7). The general conclusion that we can draw from these graphs is that the distribution $P\left(\left|v_{n}\right|\right)$ crosses over from being close to Gaussian at small $k_{n}$ to ones that fall more and more slowly with increasing $k_{n}$. This is in accord with the data of Katsuyama et al (1994) (note our wavenumber $k_{n}$ is the analogue of their midband frequency $f_{c}$ ). These graphs also illustrate that, with increasing $\operatorname{Re}_{\lambda}$, the extent of the inertial range increases and that the deviations from Gaussian distributions is far more in the dissipation range than in the inertial range. Direct plots of $\log \Gamma_{2 p}$ versus $\operatorname{Re}_{\lambda}$ are in general agreement with these statements, but are not very smooth (like our plots of $\delta \zeta_{p}$ versus $\operatorname{Re}_{\lambda}$ (figure 12)) since we have studied only eight widely separated values of $\operatorname{Re}_{\lambda}$. 


\section{Homogeneous isotropic turbulence}

\section{Concluding remarks}

We have provided a brief overview of experimental and numerical studies of coherent structures, structure functions, and probability distributions in turbulent flows that are, to a good approximation, homogeneous and isotropic. We have also outlined some of the phenomenological and stochastic models that have been used to develop an understanding of these quantities. However, as is well known, a complete theoretical understanding of homogeneous, isotropic turbulence has remained an elusive goal, in spite of the substantial progress that has been made in all the areas described above.

Our emphasis here has been on using direct numerical studies of the deterministically forced NS equation and the GOY shell model in conjunction with each other. In particular, we have explored, via ESS and GESS, an apparently universal crossover from inertial- to dissipation-range behaviours in these models. Thus our study adds to the growing evidence for similar multiscaling in NS and GOY models. It is tempting to say, therefore, that the GOY model captures some of the universal scaling properties of fluid turbulence because all known conservation laws have been built in by a suitable choice of parameters (see above). However, we must temper this optimistic view for there are many features, which seem important for fluid turbulence, that are not contained in, or poorly represented by, the GOY model. As noted by Kadanoff et al [84], there is no analogue of sweeping effects in the GOY model. Furthermore, since the GOY model has only scalar velocities and $k^{\prime}$ s which are logarithmically spaced, it cannot represent well the highvorticity filamentary structures, which are believed to be important in fully developed turbulence. Recall that the arguments which lead to the She-Leveque formula rely on the filamentary nature of these structures; yet, strangely enough, the GOY-model exponents $\zeta_{p}$ agree reasonably well with this formula. Are all these apparently important effects, which are not contained in the GOY shell model, irrelevant in some way? Unfortunately there is no clear answer to this question at this moment. Further studies are needed to elucidate the similarities and differences between the statistical properties of the solutions of the GOY shell model and the $3 d$ NS equation. However, given that numerical studies of the GOY shell model are clearly far easier than direct numerical simulations of the $3 d$ NS equation, the former can be used (with caution) as preliminary testing grounds for new theoretical ideas.

\section{Acknowledgements}

The authors thank C Jayaprakash, R Narasimha and S Ramaswamy for discussions and the referee for helpful suggestions. They also thank CSIR and BRNS, India for support and SERC, Bangalore for computational resources.

\section{References}

[1] M Van Dyke, An album of fluid motion (The Parabolic Press, Stanford, California, 1982)

[2] K R Sreenivasan and R A Antonia, Ann. Rev. Fluid Mech. 29, 435 (1997)

[3] E D Siggia, Ann. Rev. Fluid Mech. 26, 137 (1994)

[4] M Nelkin, Adv. Phys. 43, 143 (1994)

[5] V L'vov and I Procaccia, Phys. World 35, (1996)

Pramana - J. Phys., Vol. 48, No. 1, January 1997 (Part I) 


\section{Sujan $K$ Dhar et al}

[6] W D McComb, The physics of fiuid turbulence, (Oxford University Press, Oxford, 1991)

[7] U Frisch, Turbulence: the legacy of $A N$ Kolmogorov (Cambridge University Press, Cambridge, 1995)

[8] T Bohr, M H Jensen, G Paladin, A Vulpiani, Dynamical systems approach to turbulence, to be published (Cambridge University Press, Cambridge, 1997)

[9] G K Batchelor, The theory of homogeneous turbulence (Cambridge University Press, Cambridge, 1953)

[10] $\mathrm{H}$ Tennekes and J L Lumley, A first course in turbulence (MIT Press, Cambridge, Massachusetts, 1972)

[11] A S Monin and A M Yaglom, Statistical fluid mechanics (MIT Press, Cambridge, Massachusetts, 1975)

[12] G I Taylor, Proc. R. Soc. (London) A151, 421 (1935)

[13] G S Saddoughi and S V Veeravalli, J. Fluid Mech. 268, 333 (1994)

[14] $\mathrm{N}$ Goldenfeld, Lectures on phase transitions and the renormalization group (AddisonWesley, New York, 1992)

[15] To the best of our knowledge, a direct mapping of a deterministic partial differential equation (PDE) with spatiotemporal chaos onto a stochastic PDE has been carried out only for the Kuramoto-Sivashinsky (KS) equation. This mapping uses a numerical coarse-graining procedure and shows, both in one and two spatial dimensions, that the KS equation is in the universality class of the Kardar-Parisi-Zhang (KPZ) equation, i.e., the long-distance and long-time behaviours of their correlations functions are the same. (See: S Zaleski, Physica D34, 427 (1989); F Hayot, C Jayaprakash and Ch Josserand, Phys. Rev. E47, 911 (1993); C Jayaprakash, F Hayot and R Pandit, Phys. Rev. Lett. 71, 15 (1993).) The case of the deterministically forced Navier-Stokes equation is considerably more subtle; however, in the absence of a direct mapping, it has been conjectured that the appropriate PDE is the NS equation with an additive, Gaussian white noise whose variance has a power-law dependence on the wave-vector $[16,17,18]$

[16] C DeDominicis and P C Martin, Phys. Rev. A19, 419 (1979)

[17] V Yakhot and S A Orszag, J. Sci. Comput. 1, 3 (1986); Phys. Rev. Lett. 57, 1722 (1986)

[18] J K Bhattacharjee, J. Phys. A21, L 551 (1988); Phys. Rev. A40, 6374 (1989); Phys. Fluids A3, 879 (1991)

[19] C-Y Mou and P B Weichman, Phys. Rev. Lett. 70, 1101 (1993)

[20] J K Bhattacharjee, Pramana - J. Phys. 48, 365 (1997)

[21] P Bak, C Tang and K Wiesenfeld, Phys. Rev. Lett. 59, 381 (1987); Phys. Rev. A38, 364 (1988)

[22] G Stolovitzky and K R Sreenivasan, Phys. Rev. E48, R33 (1993)

[23] S Douady, Y Couder and M E Brachet, Phys. Rev. Lett. 67, 983 (1991)

[24] O Cadot, S Douady and Y Couder, Phys. Fluids 7, 630 (1995)

[25] E Villermaux, E Sixou and Y Gagne, Phys. Fluids 7, 2008 (1995)

[26] L F Richardson, Weather prediction by numerical process (Cambridge University Press, Cambridge, 1922)

[27] A N Kolmogorov, C.R. Acad. Sci. USSR 30, 301 (1941)

[28] N Cao, S Chen and K R Sreenivasan, Phys. Rev. Lett. 77, 3799 (1996)

[29] H L Grant, R W Stewart and A Moilliet, J. Fluid Mech. 12, 241 (1962)

[30] K R Sreenivasan, Phys. Fluids 7, 2778 (1995)

[31] F Anselmet, Y Gagne, E J Hopfinger and R A Antonia, J. Fluid Mech. 140, 63 (1984)

[32] R Benzi, S Ciliberto, R Trippiccione, C Baudet, F Massaioli and S Succi, Phys. Rev. E48, R29 (1993)

[33] J Herweijer and W van de Water, Phys. Rev. Lett. 74, 4651 (1995)

[34] T Katsuyama, Y Horiuchi and K Nagata, Phys. Rev. E49, 4052 (1994)

[35] S Grossman, D Lohse, V L'vov and I Procaccia, Phys. Rev. Lett. 73, 432 (1994)

[36] V S L'vov and I Procaccia, Phys. Rev. Lett. 74, 2690 (1994)

[37] G I Barenblatt and N Goldenfeld, Phys. Fluids 7, 3078 (1995)

[38] G Zocchi, J Maurer, P Tabeling and H Williame, Phys. Rev. E50, 3693 (1994)

[39] B Chabaud, A Naert, J Peinke, F Chilla, B Castaing and B Hebral, Phys. Rev. Lett. 73, 3227 (1994) 
[40] V Emsellem, L P Kadanoff, D Lohse, P Tabeling and J Wang, chao-dyn/9604009, Phys. Rev. $E$ to appear

[41] A N Kolmogorov, J. Fluid Mech. 13, 82 (1962)

[42] A A Praskovsky, Phys. Fluids A4, 2589 (1992)

[43] G Stolovitzky, P Kailasnath and K R Sreenivasan, J. Fluid Mech. 297, 275 (1995) G Stolovitzky and K R Sreenivasan, Rev. Mod. Phys. 66, 229 (1994)

[44] S T Thoroddsen and C W Van Atta, Phys. Fluids A4, 2592 (1992)

[45] Y Gagne, M Marchand and B Castaing, J. Phys. 4, 1 (1994)

[46] S Chen, G D Doolen, R H Kraichnan and L P Wang, Phys. Rev. Lett. 74, 1755 (1995)

[47] K R Sreenivasan and P Kailasnath, Phys. Fluids A5, 512 (1993)

[48] U Frisch, P L Sulem and M Nelkin, J. Fluid Mech. 87, 719 (1978)

[49] G Parisi and U Frisch, in Turbulence and predictability in geophysical fluid dynamics edited by $M$ Ghil, R Benzi and G Parisi (North-Holland, Amsterdam, 1985) pp. 84-87

[50] C Meneveau and K R Sreenivasan, J. Fluid Mech. 224, 429 (1991)

[51] Z S She and E Leveque, Phys. Rev. Lett. 72, 336 (1994)

[52] E D Siggia, J. Fluid Mech. 107, 375 (1981)

[53] Z S She, E Jackson and S A Orszag, Nature (London) 344, 226 (1990)

[54] G R Chavarria, C Baudet and S Ciliberto, Phys. Rev. Lett. 74, 1986 (1995)

[55] B Dubrulle, Phys. Rev. Lett. 73, 959 (1994)

[56] D Segel, V L'vov and I Procaccia, Phys. Rev. Lett. 76, 1828 (1996)

[57] C Meneveau, Phys. Rev. E54, 3657 (1996)

[58] R Benzi, L Biferale, S Ciliberto, M Struglia and R Tripiccione, Europhys. Lett. 32, 709 (1995)

[59] S Chen, G Doolen, J R Herring, R H Kraichnan, S A Orszag and Z S She, Phys. Rev. Lett. 70, 3051 (1993)

S Chen, G D Doolen, R H Kraichnan and Z S She, Phys. Fluids A5, 458 (1993)

[60] R H Kraichnan, J. Fluid Mech. 5, 497 (1959)

[61] D Lohse and A Müller-Groeling, Phys. Rev. Lett. 74, 1747 (1995); Phys. Rev. E54, (1996)

[62] A Praskovsky and S Oncley, Phys. Rev. Lett. 73, 3399 (1994)

[63] R Benzi, L Biferale, G Paladin, A Vulpiani and M Vergassola, Phys. Rev. Lett. 67, 2299 (1991)

[64] Z S She, Phys. Rev. Lett. 66, 600 (1991)

[65] P Kailasnath, K R Sreenivasan and G Stolovitzky, Phys. Rev. Lett. 68, 2766 (1992)

[66] G Stolovitzky, The statistical order of small scale turbulence, Ph.D. Thesis, Yale University, USA (1994), cited in ref. [2]

[67] Z S She and E C Waymire, Phys. Rev. Lett. 74, 262 (1995)

[68] S A Orszag and G S Patterson, Phys. Rev. Lett. 28, 76 (1972)

[69] R M Kerr, J. Fluid Mech. 153, 31 (1985)

[70] M M Rogers and P Moin, J. Fluid Mech. 176, 33 (1987)

[71] K Yamamoto and I Hosokawa, J. Phys. Soc. Jpn. 57, 1532 (1988)

[72] R H Kraichnan and R Panda, Phys. Fluids 31, (1988)

[73] M Meneguzzi and A Vincent, in Advances in turbulence 3 edited by A V Johansson and P H Alfredsson (Springer, Berlin, 1991) pp. 211-220; A Vincent and M Meneguzzi, J. Fluid Mech. 258, (1994)

[74] S Kida and K Ohkitani, Phys. Fluids A4, 1018 (1992)

[75] J Jimenez, A A Wray, P G Saffman and R S Rogallo, J. Fluid Mech. 255, 65 (1993)

[76] N Cao, S Chen, and Z-S She, Phys. Rev. Lett. 77, 3711 (1996)

[77] E B Gledzer, Sov. Phys. Dokl. 18, 216 (1973)

[78] K Ohkitani and M Yamada, Prog. Theor. Phys. 81, 329 (1989)

[79] A M Obukhov, Atmos. Oceanic Phys. 7, 41 (1971)

[80] V N Desnyansky and E A Novikov, Atmos. Oceanic Phys. 10, 127 (1974)

[81] M Yamada and K Ohkitani, J. Phys. Soc. Jpn. 56, 4210 (1987)

[82] M H Jensen, G Paladin and A Vulpiani, Phys. Rev. A43, 798 (1991)

[83] D Pisarenko, L Bieferale, D Courvoisier, U Frisch and M Vergassola, Phys. Fluids A5, 2533 (1993) 
[84] L Kadanoff, D Lohse, J Wang and R Benzi, Phys. Fluids 7, 617 (1995)

[85] Sujan K Dhar, Ph.D. thesis, Indian Institute of Science, Bangalore (1996) unpublished

[86] L Kadanoff, D Lohse and N Schörghofer, Physica D160, 165 (1997)

[87] L Biferale, A Lambert, R Lima and G Paladin, Physica D80, 105 (1995)

[88] Ohkitani and Yamada [79] have shown that the quadratic invariant $\sum\left(-k_{n}\right)^{\alpha}\left|v_{n}\right|^{2}$ of order $\alpha$ is conserved in the inviscid, unforced GOY shell model provided one chooses $\delta=1-\lambda^{-\alpha}$. If the invariant of order $\alpha$ is conserved, then all lower-order invariants are also conserved. The choice $\alpha=1$, i.e., $\delta=1 / 2$ for $\lambda=2$ thus conserves both the GOY-model energy and helicity in the inviscid, unforced limit; $\alpha=2$, i.e., $\delta=5 / 4$ also conserves the GOY-model enstrophy in this limit also, which is of relevance in models for two-dimensional turbulence

[89] S K Dhar, A Sain and R Pandit, Phys. Rev. Lett. (to appear) (1997)

[90] L Biferale and R Kerr, Phys. Rev. E52, 61133 (1995)

[91] R Benzi, L Biferale and G Parisi, Physica D65, 163 (1993)

[92] N Schörghofer, L Kadanoff and D Lohse, Physica D88, 40 (1995)

[93] Z S She, E Jackson and S A Orszag, Proc. R. Soc. London A434, 101 (1991)

[94] M E Brachet, D I Meiron, S A Orszag, B G Nickel, R H Morf and U Frsich, J. Fluid Mech. 130, 411 (1983)

[95] J Eggers and S Grossmann, Phys Fluids A3, 1958 (1991)

[96] S Grossmann and D Lohse, Phys. Rev. E50, 2784 (1994)

[97] E Leveque and Z S She, Phys. Rev. Lett. 75, 2690 (1995)

[98] V Borue and S A Orszag, Europhys. Lett. 29, 6875 (1995)

[99] C Foias, O Manley and L Sirovich, Phys. Fluids A2, 464 (1990)

[100] L Biferale, Phys. Fluids A5, 428 (1993)

[101] J Eggers and S Grossmann, Phys. Lett. A156, 444 (1991)

[102] D Lohse, Phys. Rev. Lett. 73, 3223 (1994) 